\title{
BMS charges in polyhomogeneous spacetimes
}

\author{
Mahdi Godazgar@ ${ }^{*}$ and George Long ${ }^{\dagger}$ \\ School of Mathematical Sciences, Queen Mary University of London, \\ Mile End Road, E1 4NS London, United Kingdom
}

(Received 21 August 2020; accepted 25 August 2020; published 14 September 2020)

\begin{abstract}
We classify the asymptotic charges of a class of polyhomogeneous asymptotically flat spacetimes with finite shear, generalizing recent results on smooth asymptotically flat spacetimes. Polyhomogeneous spacetimes are a formally consistent class of spacetimes that do not satisfy the well-known peeling property. As such, they constitute a more physical class of asymptotically flat spacetimes compared to the smooth class. In particular, we establish that the generalized conserved nonlinear Newman-Penrose charges that are known to exist for such spacetimes are a subset of asymptotic BMS charges.
\end{abstract}

DOI: 10.1103/PhysRevD.102.064036

\section{INTRODUCTION}

One of the most striking results in the mathematical study of gravitational waves in general relativity is the socalled peeling property [1-3] (see, e.g., also Ref. [4]). The peeling property is a statement regarding the asymptotic behavior of the Weyl tensor components as one approaches null infinity. For a smooth asymptotically flat spacetime, the result follows from the assumed smoothness of the unphysical spacetime upon conformal compactification [5]. In Bondi coordinates [1,2], it is

$$
\begin{aligned}
C_{a b c d}= & r^{-1} C_{a b c d}^{(N)}+r^{-2} C_{a b c d}^{(I I I)}+r^{-3} C_{a b c d}^{(I I)} \\
& +r^{-4} C_{a b c d}^{(I)}+\mathcal{O}\left(r^{-5}\right)
\end{aligned}
$$

where $r$ is an affine parameter along an outgoing null geodesic. The superscripts on the Weyl tensors on the rhs denote the components of the Weyl tensor in a null basis that is used to define the Petrov type of the spacetime. Thus, the leading order term corresponds to the Weyl tensor components of Petrov type N. Given that the Weyl tensor encompasses the remaining degrees of freedom in the curvature, the peeling property can be viewed as a statement regarding the falloff behavior of isolated gravitating bodies and the radiation they emit. However, already in Ref. [3] (see footnote 27), doubts were expressed regarding the validity of the assumptions that lead to this result. Since

\footnotetext{
*m.godazgar@qmul.ac.uk

g.long@qmul.ac.uk
}

Published by the American Physical Society under the terms of the Creative Commons Attribution 4.0 International license. Further distribution of this work must maintain attribution to the author(s) and the published article's title, journal citation, and DOI. Funded by SCOAP. then, the question of the validity of the peeling property has attracted much research; see e.g., Refs. [6-16]. What is clear by now is that the smoothness assumption precludes many interesting physically relevant cases. One class of spacetimes that moves away from the smoothness assumption are those that admit a polyhomogeneous expansion [12]. These spacetimes are formally consistent with the Einstein equations and admit the BMS group as an asymptotic symmetry group [12], have a well-defined Trautman-Bondi mass parameter [17] and admit Newman-Penrose charges $[18,19]$. Importantly, they provide an example of a more realistic class of asymptotically flat spacetimes than the smooth case.

In this paper, we shall study the asymptotic BMS charges admitted by polyhomogeneous spacetimes that have a finite shear [18]. This subset of polyhomogeneous spacetimes has a slightly better falloff property at leading order compared with the most general spacetimes. We will concentrate on this large subset of polyhomogeneous spacetimes in order to make the rather involved calculations tractable. However, we are confident that the results obtained in this paper may be generalized to the full class.

In recent work, a relation has been established [20,21], in the smooth case, between Newman-Penrose charges [22], which are a set of ten conserved nonlinear charges at null infinity, and asymptotic BMS charges [23-25], which are the charges associated with the generators of the BMS algebra via the Noether theorem. Although, such a relation ought to be natural, remarkably, such a relation had not been previously found. Indeed, in order to make progress, it has been required to extend the notion of asymptotic BMS charges to include subleading charges [20] and new dual charges $[21,26]$, which have recently been derived from first principles $[27,28]$.

Our aim in this work is to extend the formalism developed in Refs. [20,21] to classify the asymptotic 
BMS charges within the class of polyhomogeneous spacetimes with finite shear. This generalization is nontrivial for two reasons: the calculational complexity increases substantially when considering polyhomogeneous spacetimes and particular features of the polyhomogeneous expansion raises interesting new questions about the nature of charges, as will become apparent below. In particular, the nature of the characteristic value problem applied to polyhomogeneous spacetimes means that nontrivial conserved BMS charges can be defined in terms of initial data that do not evolve. This is a new feature that is specific to polyhomogeneous spacetimes and compels us to reappraise what we mean by conserved BMS charges.

Our main result is to establish a relation between asymptotic BMS charges and the generalized Newman-Penrose charges discovered in Ref. [18] for polyhomogeneous spacetimes with finite shear. Generalized Newman-Penrose charges exist for the full class of polyhomogeneous spacetimes [19] and we expect this relation to also hold in the full class.

The insights gained from this study have led to a better understanding of how Newman-Penrose charges come about and the possibility of identifying conserved charges at lower orders. An as of yet open question is whether conserved charges could exist at lower orders in the $1 / r$ expansion. We hope to tackle this interesting problem in a future work.

In Sec. II, we give some prerequisite information regarding polyhomogeneous spacetimes and the falloff behavior of their Weyl tensor components, the Einstein equations and the action of the BMS group on the metric components. Also, we define the subclass of polyhomogeneous spacetimes with finite shear. In Sec. III, we classify the standard BMS charges up to order $1 / r^{3}$ and identify a subset of five conserved nonlinear charges. Similarly, in Sec. IV, we classify the dual charges defined in Ref. [21] up to order $1 / r^{3}$ and, again, discover a subset of five conserved nonlinear charges. In Sec. V, we show, via a translation to the Newman-Penrose formalism, that the set of ten conserved charges found in Secs. III and IV is equivalent to the generalized Newman-Penrose charges of Ref. [18].

\section{PRELIMINARIES}

A polyhomogeneous spacetime is one for which the metric components can be expanded asymptotically as a combination of powers of $r^{-1}$ and positive powers of $z \equiv$ $\log r$ as $r \rightarrow \infty$. For example, a function $f$ admits a polyhomogeneous expansion if

$$
f(r)=f_{0}+\frac{f_{1}(z)}{r}+\frac{f_{2}(z)}{r^{2}}+\frac{f_{3}(z)}{r^{3}}+\cdots,
$$

where each $f_{i}$ is itself a series expansion in positive powers of $z$. As in Refs. [12,19], we restrict our attention to spacetimes where only finite powers of $z$ appear in the series, so that $f_{i}$ are polynomials in $z .{ }^{1}$ Following Ref. [19], we denote the degree of a polynomial $f$ as $\# f$.

Working with the Bondi definition of asymptotic flatness [1,2], we introduce Bondi coordinates $\left(u, r, x^{I}\right)$ with $x^{I}=\{\theta, \phi\}$, such that the metric takes the form

$$
\begin{aligned}
d s^{2}= & -F e^{2 \beta} d u^{2}-2 e^{2 \beta} d u d r \\
& +r^{2} h_{I J}\left(d x^{I}-C^{I} d u\right)\left(d x^{J}-C^{J} d u\right),
\end{aligned}
$$

where a residual gauge freedom in defining $r$ is fixed by imposing

$$
\operatorname{det} h=\operatorname{det} \omega
$$

with $\omega_{I J}$ the standard metric on the round 2-sphere. This condition implies that $h_{I J}$ has two degrees of freedom.

The Bondi definition of asymptotic flatness and the condition of polyhomogeneity requires that the metric parameters have the following large $r$ asymptotic form ${ }^{2}$ :

$$
\begin{aligned}
\beta\left(u, r, x^{I}\right)= & \frac{\beta_{0}\left(z, u, x^{I}\right)}{r^{2}}+\frac{\beta_{1}\left(z, u, x^{I}\right)}{r^{3}} \\
& +\frac{\beta_{2}\left(z, u, x^{I}\right)}{r^{4}}+o\left(r^{-4}\right), \\
F\left(u, r, x^{I}\right)= & 1+\frac{F_{0}\left(z, u, x^{I}\right)}{r}+\frac{F_{1}\left(z, u, x^{I}\right)}{r^{2}} \\
& +\frac{F_{2}\left(z, u, x^{I}\right)}{r^{3}}+\frac{F_{3}\left(z, u, x^{I}\right)}{r^{4}}+o\left(r^{-4}\right), \\
C^{I}\left(u, r, x^{I}\right)= & \frac{C_{0}^{I}\left(z, u, x^{I}\right)}{r^{2}}+\frac{C_{1}^{I}\left(z, u, x^{I}\right)}{r^{3}}+\frac{C_{2}^{I}\left(z, u, x^{I}\right)}{r^{4}} \\
& +\frac{C_{3}^{I}\left(z, u, x^{I}\right)}{r^{5}}+o\left(r^{-5}\right), \\
h_{I J}\left(u, r, x^{I}\right)= & \omega_{I J}+\frac{B_{I J}\left(z, u, x^{I}\right)}{r}+\frac{\bar{C}_{I J}\left(z, u, x^{I}\right)}{r^{2}} \\
& +\frac{\bar{D}_{I J}\left(z, u, x^{I}\right)}{r^{3}}+\frac{\bar{E}_{I J}\left(z, u, x^{I}\right)}{r^{4}}+o\left(r^{-4}\right),
\end{aligned}
$$

where

$$
\begin{aligned}
& \bar{C}_{I J}=C_{I J}+\frac{1}{4} B^{2} \omega_{I J}, \quad \bar{D}_{I J}=D_{I J}+\frac{1}{2} B_{K L} C^{K L} \omega_{I J} \\
& \bar{E}_{I J}=E_{I J}+\left(\frac{1}{2} B_{K L} D^{K L}+\frac{1}{4} C^{2}-\frac{1}{32}\left(B^{2}\right)^{2}\right) \omega_{I J}
\end{aligned}
$$

\footnotetext{
${ }^{1}$ Relaxing this condition would mean that the infinite series in $z$ that would appear in these calculations would in fact reduce to integer powers of $r$. Therefore, our analysis, which treats the expansions in $1 / r$ and $z$ independently, would no longer be valid.

${ }^{2}$ Note the slight difference in notation in the expansion of $h_{I J}$ compared to Refs. [20,21], cf. Eq. (2.2) of Ref. [20].
} 
with $B^{2}=B_{I J} B^{I J}$ and $C^{2}=C_{I J} C^{I J}$. This form of the $h_{I J}$ expansion is chosen so that condition (2.3) simply translates to

$$
\operatorname{Tr} B=\operatorname{Tr} C=\operatorname{Tr} D=\operatorname{Tr} E=0
$$

at this order, where for some tensor $X_{I J}, \operatorname{Tr} X \equiv \omega^{I J} X_{I J}$. Furthermore, the 2-sphere tensors $B, C, D$ and $E$ are independent and parametrize the 2 degrees of freedom of $h_{I J}$ at each order.

\section{A. Asymptotic behavior of Weyl scalar $\Psi_{0}$}

For spacetimes that are analytic in $1 / r$, i.e., there exist no $\log$ terms, the Weyl tensor satisfies the so-called peeling property [3], which can be simply stated as the fact that the Weyl tensor in the unphysical spacetime vanishes at null infinity. In Newman-Penrose language [3], this statement is equivalent to the fact that

$$
\Psi_{i}=O\left(\frac{1}{r^{5-i}}\right), \quad i \in\{0, \ldots, 4\}
$$

where the $\Psi_{i}$ are the Newman-Penrose Weyl scalars defined with respect to a complex null frame $\left(\ell^{a}, n^{a}, m^{a}, \bar{m}^{a}\right)$,

$$
\begin{array}{ll}
\Psi_{0}=\ell^{a} m^{b} \ell^{c} m^{d} C_{a b c d}, & \Psi_{1}=\ell^{a} n^{b} \ell^{c} m^{d} C_{a b c d}, \\
\Psi_{2}=\ell^{a} m^{b} \bar{m}^{c} n^{d} C_{a b c d}, & \Psi_{3}=\ell^{a} n^{b} \bar{m}^{c} n^{d} C_{a b c d}, \\
\Psi_{4}=n^{a} \bar{m}^{b} n^{c} \bar{m}^{d} C_{a b c d} . &
\end{array}
$$

As we shall explain below, the peeling property no longer holds in polyhomogeneous spacetimes [12,29]. Moreover, we shall find that the Weyl scalar $\Psi_{0}$ falls off too slowly. This will lead us to make some further assumptions on the metric expansion (2.4).

We begin by choosing a complex null frame $e_{\mu}{ }^{a}=$ $\left(\ell^{a}, n^{a}, m^{a}, \bar{m}^{a}\right)$ with inverse $E^{\mu}{ }_{a}$,

$$
\begin{aligned}
& g_{a b}=E^{\mu}{ }_{a} E^{\nu}{ }_{b} \eta_{\mu \nu}, \\
& \eta_{\mu \nu}=\left(\begin{array}{cccccc}
0 & & -1 & & & \\
& & & & \mathbf{0} & \\
-1 & & 0 & & \\
& & & 0 & & 1 \\
& \mathbf{0} & & & \\
& & & 1 & & 0
\end{array}\right)
\end{aligned}
$$

where

$$
\begin{aligned}
\ell & =\frac{\partial}{\partial r}, \quad n=e^{-2 \beta}\left[\frac{\partial}{\partial u}-\frac{1}{2} F \frac{\partial}{\partial r}+C^{I} \frac{\partial}{\partial x^{I}}\right] \\
m & =\frac{\hat{m}^{I}}{r} \frac{\partial}{\partial x^{I}}, \quad \ell^{b}=-e^{2 \beta} d u, \quad n^{b}=-\left(d r+\frac{1}{2} F d u\right) \\
m^{b} & =r \hat{m}_{I}\left(d x^{I}-C^{I} d u\right),
\end{aligned}
$$

$$
2 \hat{m}^{(I} \overline{\hat{m}}^{J)}=h^{I J}
$$

with $h^{I J}$ the matrix inverse of $h_{I J}$. The polyhomogeneous expansion (2.4) implies that

$$
\Psi_{0} \sim \frac{1}{r^{3}}\left(\mathcal{B}_{\theta \theta}-\frac{i}{\sin \theta} \mathcal{B}_{\theta \phi}\right)+\mathcal{O}\left(r^{-4} \log ^{N_{4}} r\right),
$$

where $\mathcal{B}_{I J}=\partial_{z} B_{I J}-\partial_{z}^{2} B_{I J}$. Compare this with the falloff of $\Psi_{0}$ in Refs. [20-22]:

$$
\Psi_{0}=\frac{\Psi_{0}^{5}}{r^{5}}+\frac{\Psi_{0}^{6}}{r^{6}}+\frac{\Psi_{0}^{7}}{r^{7}}+o\left(r^{-7}\right)
$$

In this paper, in order to make progress, we will assume that $\Psi_{0}$ behaves asymptotically as $\mathcal{O}\left(r^{-4} \log ^{N_{4}} r\right)$. While, it is true that Newman-Penrose charges exist more generally for any polyhomogeneous spacetime defined by the falloffs (2.4) [19], the analysis is much simpler if we assume that the leading order term in the shear of the null congruence defined by $\ell$ has no $\log$ terms [18]. This is equivalent to the requirement that $\Psi_{0} \sim \mathcal{O}\left(r^{-4} \log ^{N_{4}} r\right)$, or that

$$
\mathcal{B}_{\theta \theta}-\frac{i}{\sin \theta} \mathcal{B}_{\theta \phi}=0
$$

which is equivalent to the condition that $\mathcal{B}_{\theta \theta}=\mathcal{B}_{\theta \phi}=0$, given that $\mathcal{B}_{I J}$ is real. From the fact that $B_{I J}$ is traceless and symmetric, we deduce that $\mathcal{B}_{I J}$ is traceless and symmetric and hence the above condition is equivalent to

$$
\mathcal{B}_{I J}=\partial_{z} B_{I J}-\partial_{z}^{2} B_{I J}=0 .
$$

The fact that $B_{I J}$ is a polynomial in $z$ of finite order implies that

$$
\partial_{z} B_{I J}=0
$$

i.e., that $B_{I J}$ is independent of $z$ and contains no log terms. Henceforth, we shall assume that this condition always holds. We shall find below that, together with this condition, the Einstein equations imply that all leading order terms in (2.4) are independent of $z .^{3}$

\footnotetext{
${ }^{3}$ At the next order, $\Psi_{0} \sim \frac{1}{r^{4}}\left(\mathcal{C}_{\theta \theta}+\frac{i}{\sin \theta} \mathcal{C}_{\theta \phi}\right)+\mathcal{O}\left(r^{-5} \log ^{N_{5}} r\right)$, where $\mathcal{C}_{I J}=-2 C_{I J}+3 \partial_{z} C_{I J}^{r}-\partial_{z}^{2} C_{I J}$. Requiring that $\Psi_{0}=$ $o\left(r^{-5}\right)$ would imply that $C_{I J}=0$, which recovers the falloff conditions (2.2) in Ref. [20].
} 


\section{B. Notation}

For brevity, it will prove useful to use the following notation:

$$
\int^{\lambda} X(z) \equiv e^{\lambda z} \int d z e^{-\lambda z} X(z)
$$

for $\lambda$ an integer, in order to reduce the size of some of the equations. Furthermore, $\int^{\lambda}$ will be treated as an operator acting on the right, so we have for example

$$
\begin{aligned}
\left(3 \int^{4}-\right. & \left.2 \int^{1}+\int^{0}+6-\partial_{z}\right) X(z) \\
= & 3 e^{4 z} \int e^{-4 z} X(z) d z-2 e^{z} \int e^{-z} X(z) d z \\
& +\int X(z) d z+6 X(z)-X^{\prime}(z) .
\end{aligned}
$$

For $\lambda \neq 0, \int^{\lambda}$ does not change the order of the polynomial in $z$; see Appendix A. However, $\partial_{z}$ decreases the order by 1 and $\int^{0}$ increases it by 1 .

Moreover, angled brackets \langle\rangle on pairs of indices will be used to denote the symmetric trace-free part; thus, for an arbitrary tensor $X_{I J}$

$$
X_{\langle I J\rangle} \equiv \frac{1}{2}\left(X_{I J}+X_{J I}-\omega^{K L} X_{K L} \omega_{I J}\right)
$$

For example,

$$
\begin{aligned}
B_{\langle I| K} C^{K}{ }_{|J\rangle}= & \frac{1}{2} B_{I K} C^{K}{ }_{J}+\frac{1}{2} B_{J K} C^{K}{ }_{I} \\
& -\frac{1}{2} B_{K L} C^{K L} \omega_{I J} .
\end{aligned}
$$

\section{Einstein equations}

We will assume that the energy-momentum tensor satisfies the falloff conditions ${ }^{4}$

$T_{00}=o\left(r^{-4}\right), \quad T_{0 m}=o\left(r^{-3}\right), \quad T_{01}=o\left(r^{-3}\right)$.

The Einstein equation then yields

$$
\begin{gathered}
G_{00}=o\left(r^{-4}\right) \Rightarrow \beta_{0}=-\frac{1}{32} B^{2}, \\
G_{0 m}=o\left(r^{-3}\right) \Rightarrow C_{0}^{I}=-\frac{1}{2} D_{J} B^{I J}, \\
G_{01}=o\left(r^{-3}\right) \Rightarrow \partial_{z} F_{0}=0,
\end{gathered}
$$

where $D_{I}$ is the standard covariant derivative associated with the round-sphere metric $\omega_{I J}$. Since $B_{I J}$ is independent of $z$, we conclude the leading order terms in (2.4) are all independent of $z$.

Assuming stronger falloff conditions for the energy momentum tensor, the Einstein equations imply the following:

$$
\begin{gathered}
G_{00}=o\left(r^{-5}\right) \Rightarrow \beta_{1}=-\frac{1}{8}\left(\int^{3}+1\right) B_{I J} C^{I J} \\
G_{00}=o\left(r^{-6}\right) \Rightarrow \beta_{2}=-\frac{1}{8}\left(\int^{4}+1\right) B_{I J} D^{I J}-\frac{1}{8}\left(2 \int^{4}+1\right) C^{2}+\frac{1}{16} \int^{4} \partial_{z} C_{I J} \partial_{z} C^{I J}+\frac{1}{128}\left(B^{2}\right)^{2} \\
G_{0 m}=o\left(r^{-4}\right) \Rightarrow C_{1}^{I}=\left(C_{1}^{0}\right)^{I}-\frac{1}{3}\left(\int^{3}+2 \int^{0}\right) D_{J} C^{I J} \\
G_{0 m}=o\left(r^{-5}\right) \Rightarrow C_{2}^{I}=-\frac{3}{4} B^{I J}\left(C_{1}^{0}\right)_{J}-\frac{1}{3}\left(\int^{4}+2 \int\right) D_{J}^{1} D^{I J}-\frac{1}{2}\left(\int^{4}-\int^{0}\right) B^{I J} D^{K} C_{J K}+\frac{1}{4}\left(2 \int^{4}-\int^{3}\right) B_{J K} D^{I} C^{J K}-\frac{1}{4} \int C^{J K} D^{I} B_{J K} \\
+\frac{1}{64} B^{2} D_{J} B^{I J}-\frac{1}{16} B^{I J} D_{J} B^{2}
\end{gathered}
$$

${ }^{4}$ Given some arbitrary vector $V_{a}$, we denote the components in the null basis as follows:

$$
\ell^{a} V_{a} \equiv V_{0}=-V^{1}, \quad n^{a} V_{a} \equiv V_{1}=-V^{0}, \quad m^{a} V_{a} \equiv V_{m}=V^{\bar{m}},
$$

with the obvious generalization to tensors. 


$$
\begin{aligned}
& G_{0 m}=o\left(r^{-6}\right) \Rightarrow C_{3}^{I}=\frac{9}{80} B^{2}\left(C_{1}^{0}\right)^{I}+3 \int^{5}\left(C_{1}^{0}\right)_{J} C^{I J}-\frac{1}{3}\left(\int^{5}+22^{2}\right) D_{J} E^{I J}-\frac{1}{4} \int^{4} D^{J K} D^{I} B_{J K}+\frac{1}{4}\left(2 \int^{5}-\int^{4}\right) B_{J K} D^{K} D^{I J} \\
& -\frac{1}{4}\left(\int^{4}-2 \int^{1}\right) B^{I J} D^{K} D_{J K}+\frac{1}{2}\left(\int^{5}-\int^{4}\right) D^{I} C^{2}-\frac{1}{24}\left(\int^{5}-3 \int^{4}+2 \int^{2}\right) D^{I}\left(\partial_{z} C^{J K} \partial_{z} C_{J K}\right) \\
& -\frac{1}{6}\left(\int^{5}-\int^{2}\right) D^{I} C^{J K} \partial_{z} C_{J K}+\frac{2}{3}\left(\int^{5}-\int^{2}\right) \partial_{z} C^{I J} D^{K} C_{J K}-\frac{2}{3}\left(\int^{5}-\int^{2}\right) C^{I J} D^{K} C_{J K}-2 \int^{5}\left(C^{I J} \int d z D^{K} C_{J K}\right) \\
& +\frac{1}{8}\left(5^{5}+\int^{3}\right) B^{I J} C^{K L} D_{J} B_{K L}-\frac{1}{8}\left(3 \int^{5}-\int^{3}\right) B^{K L} C_{K L} D_{J} B^{I J}+\frac{1}{8}\left(\int^{5}+\int^{3}\right) B^{I J} B^{K L} D_{J} C_{K L} \\
& -\frac{1}{240}\left(7 \int^{5}-40 \int^{2}+18 \int^{0}\right) B^{2} D_{J} C^{I J}-\frac{1}{12}\left(5 \int^{5}-2 \int^{2}\right) C^{I J} D_{J} B^{2}+\frac{3}{160} B^{2} D^{I} B^{2}, \\
& G_{01}=o\left(r^{-4}\right) \Rightarrow F_{1}=-\frac{1}{2} D_{I}\left(C_{1}^{0}\right)^{I}-\frac{1}{3}\left(\int^{3}-\int^{0}\right) D_{I} D_{J} C^{I J}+\frac{1}{2} D_{I} B^{I J} D^{K} B_{J K}-\frac{1}{8} D_{I} B_{J K} D^{I} B^{J K}+\frac{3}{32}(\square-2) B^{2}, \\
& G_{01}=o\left(r^{-5}\right) \Rightarrow F_{2}=-\frac{3}{4}\left(C_{1}^{0}\right)^{I} D^{J} B_{I J}-\frac{1}{3}\left(\int^{4}-\int^{1}\right) D_{I} D_{J} D^{I J}+\frac{1}{4}\left(\int^{4}-\int^{3}\right) \square\left(B_{I J} C^{I J}\right)-\frac{1}{4} \int^{4} C^{I J} \square B_{I J}+\frac{1}{4}\left(2 \int^{4}-\int^{3}\right) B_{I J} C^{I J} \\
& -\frac{1}{2}\left(\int^{4}-\int^{0}\right) D_{I} B^{I J} D^{K} C_{J K}+\frac{1}{32} B^{I J} B^{K L} D_{K} D_{L} B_{I J}+\frac{1}{64} B^{2} D_{I} D_{J} B^{I J}-\frac{1}{32} B^{I J} D_{I} B_{K L} D_{J} B^{K L} \\
& +\frac{5}{64} D^{I} B^{2} D^{J} B_{I J}
\end{aligned}
$$

$$
\begin{aligned}
G_{01}=o\left(r^{-6}\right) \Rightarrow F_{3}= & \frac{3}{4}\left(C_{1}^{0}\right)^{I}\left(C_{1}^{0}\right)_{I}+\frac{3}{160} D_{I}\left(B^{2}\left(C_{1}^{0}\right)^{I}\right)+\left(3 \int^{5}-2 \int^{3}-\int^{0}\right)\left(C_{1}^{0}\right)^{I} D^{J} C_{I J}+\frac{3}{2}\left(2 \int^{5}-\int^{3}\right) C_{I J} D^{J}\left(C_{1}^{0}\right)^{I} \\
& -\frac{1}{3}\left(\int^{5}-\int^{2}\right) D_{I} D_{J} E^{I J}+\frac{1}{4}\left(2 \int^{5}-\int^{4}\right) B_{I J} D^{I J}+\frac{1}{4} \int^{5} B_{I J} \square D^{I J}-\frac{1}{4} \int^{4} \square\left(B_{I J} D^{I J}\right)+\frac{1}{2} \int^{1} D_{I} B_{J K} D^{I} D^{J K}
\end{aligned}
$$$$
+\frac{1}{2}\left(\int^{5}-\int^{1}\right) D_{I} B_{J K} D^{J} D^{I K}+\frac{1}{12}\left(4 \int^{5}-6 \int^{4}+3 \int^{3}-\int^{2}\right) \square C^{2}
$$$$
-\frac{1}{24}\left(\int^{5}-3 \int^{4}+3 \int^{3}-\int^{2}\right) \square\left(\partial_{z} C_{I J} \partial_{z} C^{I J}\right)+\frac{1}{12}\left(2 \int^{5}-3 \int^{3}+\rho^{2}\right)\left(\partial_{z} C_{I J} \square C^{I J}\right)
$$$$
-\frac{1}{2}\left(2 \int^{5}-\int^{3}\right)\left(C_{I J} \int d z \square C^{I J}\right)-\frac{1}{2}\left(2 \int^{5}+\int^{4}-2 \int^{3}\right) C^{2}+\frac{1}{8}\left(\int^{4}-\int^{3}\right)\left(\partial_{z} C_{I J} \partial_{z} C^{I J}\right)+\left(2 \int^{5}-\int^{3}\right)\left(C_{I J} \int d z C^{I J}\right)
$$$$
-\frac{1}{12}\left(\int^{5}-\int^{2}\right) D_{I} C_{J K} D^{I} C^{J K}+\left(\int^{5}-\int^{3}\right) D^{I} C_{I J} D_{K} C^{J K}
$$$$
-\frac{2}{3}\left(3 \int^{5}-2 \int^{3}\right)\left(D^{I} C_{I J} \int d z D_{K} C^{J K}\right)+\frac{1}{3}\left(\int d z D^{I} C_{I J}\right)\left(\int d z D_{K} C^{J K}\right)
$$$$
+\frac{1}{8}\left(5 \int^{5}-3 \int^{3}\right) B^{I J} C^{K L} D_{I} D_{J} B_{K L}-\frac{1}{96}\left(40 \int^{5}-33 \int^{3}+8 \int^{2}\right) C^{I J} D_{I} D_{J} B^{2}
$$$$
-\frac{1}{8}\left(3 \int^{5}-\int^{3}\right) B^{K L} C_{K L} D_{I} D_{J} B^{I J}+\frac{1}{8}\left(\int^{5}-\int^{3}\right) B^{I J} B^{K L} D_{I} D_{J} C_{K L}-\frac{1}{240}\left(7 \int^{5}-30^{3}+20 \int^{2}+3 \int^{0}\right) B^{2} D_{I} D_{J} C^{I J}
$$$$
-\frac{1}{240}\left(107 \int^{5}-150 \int^{3}+40 \int^{2}+3 \int^{0}\right) D_{I} C^{I J} D_{J} B^{2}+\frac{3}{4}\left(\int^{5}-\int^{3}\right) B^{I J} D_{I} B_{K L} D_{J} C^{K L}-\frac{1}{4}\left(\int^{5}-\int^{3}\right) B^{K L} D_{I} B^{I J} D_{J} C_{K L}
$$$$
-\frac{1}{8} \int^{3} C^{I J} D_{I} B_{K L} D_{J} B^{K L}+\frac{1}{4}\left(\int^{5}+\int^{3}\right) C^{K L} D_{I} B_{K L} D_{J} B^{I J}-\frac{1}{2} \int^{3} C_{K L} D_{I} B^{I K} D_{J} B^{J L}
$$$$
+\frac{5}{512}\left(B^{2}\right)^{2}-\frac{17}{5120} \square\left(B^{2}\right)^{2}+\frac{13}{1024} D_{I} B^{2} D^{I} B^{2}+\frac{3}{128} B^{2} D_{I} B_{J K} D^{I} B^{J K}-\frac{1}{32} B^{2} D_{I} B_{J K} D^{J} B^{I K},
$$$$
G_{m m}=o\left(r^{-3}\right) \Rightarrow \partial_{u} C_{I J}=0
$$ 


$$
\begin{aligned}
& G_{m m}=o\left(r^{-4}\right) \Rightarrow \partial_{u} D_{I J}=\frac{1}{8} B_{I J} \partial_{u} B^{2}-\frac{1}{4} B_{I J} F_{0}-\frac{1}{2} D_{\langle I}\left(C_{1}^{0}\right)_{J\rangle}-\frac{1}{6}\left(4 \int^{3}+2 \int^{0}+3-3 \partial_{z}\right) C_{I J}+\frac{1}{6}\left(2 \int^{3}+\int^{0}\right) \square C_{I J} \\
& -\frac{1}{8} B_{I J} D_{K} D_{L} B^{K L}+\frac{1}{32} D_{\langle I} D_{J\rangle} B^{2}+\frac{1}{2} D_{\langle I}\left(B_{J\rangle K} D_{L} B^{K L}\right)-\frac{1}{8} D_{\langle I|} B_{K L} D_{|J\rangle} B^{K L}, \\
& G_{m m}=o\left(r^{-5}\right) \Rightarrow \partial_{u} E_{I J}=\frac{1}{2} D^{K}\left(\left(C_{1}^{0}\right)_{\langle I} B_{J\rangle K}\right)+\frac{1}{2}\left({ }^{3}-1+\partial_{z}\right) C_{I J} F_{0}+\frac{1}{2} B^{K L} C_{K L} \partial_{u} B_{I J}+B_{K\langle I} C_{J\rangle L} \partial_{u} B^{K L} \\
& +\frac{1}{2} \int^{3} B_{I J} C^{K L} \partial_{u} B_{K L}-\frac{1}{4}\left(\int^{3}+1\right) C_{I J} \partial_{u} B^{2}+\frac{1}{3}\left(2 \int^{4}+\int^{1}\right) D_{\langle I} D^{K} D_{J\rangle K}-\frac{1}{2}\left(2-\partial_{z}\right) D_{I J} \\
& -\frac{1}{2}\left(\int^{3}+1\right) C^{K L} D_{K} D_{L} B_{I J}+\frac{1}{2}\left(\int^{3}+1\right) C^{K L} D_{\langle I} D_{J\rangle} B_{K L} \\
& +\frac{1}{4}\left(\int^{3}+1\right) C_{I J} D_{K} D_{L} B^{K L}-\frac{1}{6}\left(3 \int-2 \int^{3}-\int^{0}\right) B^{K L} D_{\langle I} D_{J\rangle} C_{K L} \\
& +\frac{1}{6}\left(3 \int^{4}-2 \int^{3}-\int^{0}\right) B_{I J} D_{K} D_{L} C^{K L}-\frac{1}{6}\left(3 \int^{4}-\int^{3}+\int^{0}\right) B^{K L} D_{K} D_{L} C_{I J} \\
& -\int^{4} D_{\langle I} B^{K L} D_{J\rangle} C_{K L}+\frac{1}{2}\left(2 \int^{3}+1\right) D_{K} B^{K L} D_{L} C_{I J}+\int^{4} D_{K} C^{K L} D_{L} B_{I J} \\
& -\frac{1}{3}\left(3 \int^{4}+2 \int^{3}+\int^{0}\right) D^{K} C_{K\langle I} D^{L} B_{J\rangle L}+\frac{5}{32} D^{K}\left(B^{2} D_{\langle I} B_{J\rangle K}\right)-\frac{1}{8} D^{K}\left(B_{K\langle I} D_{J\rangle} B^{2}\right), \\
& G_{11}=o\left(r^{-2}\right) \Rightarrow \partial_{u} F_{0}=-\frac{1}{2} D_{I} D_{J} \partial_{u} B^{I J}+\frac{1}{4} \partial_{u} B_{I J} \partial_{u} B^{I J}, \\
& G_{1 m}=o\left(r^{-3}\right) \Rightarrow \partial_{u}\left(C_{1}^{0}\right)^{I}=\frac{1}{3} D^{I} F_{0}+\frac{1}{6} \square D_{J} B^{I J}-\frac{1}{6} D^{I} D_{J} D_{K} B^{J K}+\frac{1}{8} B^{J K} \partial_{u} D^{I} B_{J K} \\
& +\frac{5}{8} \partial_{u} B_{J K} D^{I} B^{J K}-\frac{2}{3} \partial_{u} B_{J K} D^{K} B^{I J}-\frac{1}{6} D_{J} B^{I J},
\end{aligned}
$$

where $\left(C_{1}^{0}\right)^{I}$ has no $z$ dependence and $\square=D^{I} D_{I}$ is the covariant Laplacian on the unit 2-sphere.

The above Einstein equations are the generalizations of the Einstein equations (2.15)-(2.26) of Ref. [20]. Setting $C_{I J}=0$ and assuming that all tensors are $z$ independent, so that Eq. (A4) can be used, the above equations reduce to the respective equations in Ref. [20] by taking $B_{I J} \rightarrow C_{I J}$.

Assuming the vacuum Einstein equations to the appropriate order, it is possible to deduce the $z$ order of each metric parameter in (2.4). In general,

$$
\begin{aligned}
& \# B_{I J}=0, \quad \# C_{I J} \equiv N_{C} \geq 0, \quad \# D_{I J} \equiv N_{D}, \quad \# E_{I J} \equiv N_{E}, \\
& \# \beta_{0}=0, \quad \# \beta_{1}=N_{C}, \quad \# \beta_{2}=\max \left\{N_{D}, 2 N_{C}\right\} \\
& \# C_{0}^{I}=0, \quad \# C_{1}^{I}=N_{C}+1, \\
& \# C_{2}^{I}=N_{D}, \quad \# C_{3}^{I}=\max \left\{N_{E}, 2 N_{C}+1\right\} \\
& \# F_{0}=0, \quad \# F_{1}=N_{C}+1, \\
& \# F_{2}=N_{D}, \quad \# F_{3}=\max \left\{N_{E}, 2\left(N_{C}+1\right)\right\} .
\end{aligned}
$$

An important assumption that we shall rely upon in what follows is

$$
N_{E} \geq N_{D}>N_{C} \geq 0
$$

This is the case for generic initial data [19]. It is possible that in special cases, for example if $D_{I} C^{I J}=0$, the above assumption does not hold. Nevertheless, all the charges obtained in this paper are still conserved in such cases.

\section{BMS group}

The asymptotic symmetry group of polyhomogeneous spacetimes is given by the BMS group, as with the smooth case [12]. This group is obtained by imposing that the variation of the metric under the generators of the asymptotic symmetry group respects the form of the metric and the gauge choices. These conditions imply a group of the form

$$
\mathrm{BMS}=\mathrm{SL}(2, \mathbb{C}) \ltimes \mathrm{ST},
$$

where ST represents the infinite affine group of supertranslations parametrized by a $u$ and $r$-independent function $s\left(x^{I}\right)$ and generated by diffeomorphisms of the form

$\xi=s \partial_{u}+\int d r \frac{e^{2 \beta}}{r^{2}} h^{I J} D_{J} s \partial_{I}-\frac{r}{2}\left(D_{I} \xi^{I}-C^{I} D_{I} s\right) \partial_{r}$ 
As in Ref. [20], we shall concentrate on the supertranslation part of the BMS algebra.

We list below the variation of some of the metric components under supertranslations that will be useful later:

$$
\begin{aligned}
& \delta F_{0}=s \partial_{u} F_{0}-\frac{1}{2} \partial_{u} B^{I J} D_{I} D_{J} s-D_{J} \partial_{u} B^{I J} D_{I} s, \\
& \delta\left(C_{1}^{0}\right)^{I}=s \partial_{u}\left(C_{1}^{0}\right)^{I}+\frac{1}{16} \partial_{u} B^{2} D^{I} s+F_{0} D^{I} s-\frac{1}{4} B^{J K} D^{I} D_{J} D_{K} s-\frac{1}{2} B^{I J} D_{J} \square s \\
& +\frac{1}{2} D^{J} B^{I K} D_{J} D_{K} s-\frac{3}{4} D^{I} B^{J K} D_{J} D_{K} s-\frac{1}{2} D_{J} B^{J K} D_{K} D^{I} s \\
& -\frac{1}{2} D^{I} D^{J} B_{J K} D^{K} s+\frac{1}{2} D^{J} D_{K} B^{I K} D_{J} s-B^{I J} D_{J} s, \\
& \delta B_{I J}=s \partial_{u} B_{I J}-2 D_{\langle I} D_{J\rangle} s, \\
& \delta C_{I J}=s \partial_{u} C_{I J} \\
& \delta D_{I J}=s \partial_{u} D_{I J}-2\left(C_{1}^{0}\right)_{\langle I} D_{J\rangle} s-\frac{1}{4} B_{I J} B^{K L} D_{K} D_{L} s-\frac{1}{8} B^{2} D_{\langle I} D_{J\rangle} s+\frac{1}{8} D_{\langle I} B^{2} D_{J\rangle} s \\
& +D_{K} B^{K L} B_{L\langle I} D_{J\rangle} s-\left(2 \int^{3}+1\right) D^{K} C_{I J} D_{K} s+\frac{4}{3}\left(2 \int^{3}+\int^{0}\right) D^{K} C_{K\langle I} D_{J\rangle} s \\
& -\left(\int^{3}+1-\frac{1}{2} \partial_{z}\right) C_{I J} \square s, \\
& \delta E_{I J}=s \partial_{u} E_{I J}+\frac{1}{2}\left(C_{1}^{0}\right)_{\langle I} B_{J\rangle K} D^{K} s-\frac{1}{2}\left(2 \int^{4}+3-\partial_{z}\right) D_{I J} \square s \\
& +\frac{1}{3}\left(2 \int^{4}+4 \int^{1}-3\right) D^{K} D_{K\langle I} D_{J\rangle} s-\left(2 \int^{4}+1\right) D_{\langle I} D_{J\rangle} D^{K} s \\
& +\frac{1}{4}\left(4 \int^{3}+2-\partial_{z}\right) B^{K L} C_{I J} D_{K} D_{L} s-\frac{1}{2}\left(\int^{3}+1\right)\left(B_{I J} C^{K L} D_{K} D_{L} s+B^{K L} C_{K L} D_{\langle I} D_{J\rangle} s\right) \\
& +\int^{3}\left(C_{K L} D_{\langle I} B^{K L} D_{J\rangle} s-C^{K L} D_{K} B_{I J} D_{L} s\right)+C_{K\langle I} D_{J\rangle} B^{K L} D_{L} s+D_{K} B^{K L} C_{L\langle I} D_{J\rangle} s \\
& -\frac{1}{2} \partial_{z}\left(C_{I J} D_{K} B^{K L} D_{L} s\right)-\left(\int^{4}-\int^{3}\right) B_{K L} D_{\langle I} C^{K L} D_{J\rangle} s-2 \int^{3} B_{K\langle I} D_{J\rangle} C^{K L} D_{L} s \\
& +\frac{1}{2} B^{K L} D_{K} C_{I J} D_{L} s+\frac{1}{3}\left(3 \int^{4}+2 \int^{3}+\int^{0}\right) D_{K} C^{K L} B_{L\langle I} D_{J\rangle} s+\frac{1}{3}\left(\int^{3}-\int^{0}\right) B_{I J} D_{K} C^{K L} D_{L} s \\
& +\frac{5}{32} D^{K}\left(B^{2} B_{K\langle I} D_{J\rangle} s\right)+\frac{5}{32} B^{2} D^{K} D_{\langle I} B_{J\rangle K}-\frac{1}{8} B_{K\langle I} D_{J\rangle} B^{2} D^{K} s .
\end{aligned}
$$

These variations are guaranteed to preserve the form of the metric. However, we will impose further constraints on the metric via the Einstein equations by assuming particular falloffs of the components of the energy-momentum tensor. If we impose a particular falloff on one component, we may need to impose further conditions on other components so that the desired falloff condition is preserved under the BMS action. The variation of a particular component (for fixed $\alpha, \beta \in\{0,1, m, \bar{m}\})$ is given by

$$
\delta_{\xi} T_{\alpha \beta}=\xi^{c} \partial_{c} T_{\alpha \beta}+T_{c \beta} \partial_{\alpha} \xi^{c}+T_{c \alpha} \partial_{\beta} \xi^{c} .
$$

If we insist that $T_{\alpha \beta}=o\left(r^{-n}\right)$, certain falloff conditions must be obeyed by $T_{c \alpha}$ and $T_{c \beta}$. When assuming a particular falloff condition, we will also assume that the relevant conditions are satisfied for the other components. This can always be done and presents no issues in our calculations.

\section{STANDARD BMS CHARGES}

The asymptotic charges associated with the asymptotic BMS symmetry group are given by the following expression [24] (see also Refs. [23,30]): 


$$
\begin{aligned}
\not \mathcal{Q}_{\xi}[\delta g, g] & =\frac{1}{8 \pi G} \int_{S} \star H[\xi, g, \delta g] \\
& =\frac{1}{8 \pi G} \int_{S} d \Omega r^{2} e^{2 \beta} H^{u r}[\xi, g, \delta g],
\end{aligned}
$$

where we have used the form of the background metric of interest (2.2) in the second equality. The 2-form $H$ is given by

$$
\begin{aligned}
H= & \frac{1}{2}\left\{\xi_{b} g^{c d} \nabla_{a} \delta g_{c d}-\xi_{b} \nabla^{c} \delta g_{a c}+\xi^{c} \nabla_{b} \delta g_{c a}\right. \\
& \left.+\frac{1}{2} g^{c d} \delta g_{c d} \nabla_{b} \xi_{a}+\frac{1}{2} \delta g_{b c}\left(\nabla_{a} \xi^{c}-\nabla^{c} \xi_{a}\right)\right\} d x^{a} \wedge d x^{b} .
\end{aligned}
$$

The slash on the variational symbol $\delta$ in (3.1) signifies the fact that the variation is not, in general, integrable.

We have all the ingredients to compute charges, namely the background metric $g_{a b}$ given by (2.2) and the symmetry generators given by (2.40). Plugging the above into Eq. (3.1) leads to an expansion of the form [20]

$$
\begin{aligned}
\not \mathcal{Q}_{\xi}[\delta g, g]= & \frac{1}{16 \pi G} \int_{S} d \Omega\left\{\not \mathcal{I}_{0}+\frac{\not \mathcal{I}_{1}(z)}{r}+\frac{\phi \mathcal{I}_{2}(z)}{r^{2}}\right. \\
& \left.+\frac{\not \mathcal{I}_{3}(z)}{r^{3}}+o\left(r^{-3}\right)\right\},
\end{aligned}
$$

where each $\not \mathcal{I}_{i}(z)$ is a polynomial of finite order in $z=\log r$. The first term $\not \mathcal{I}_{0}$ in the expansion above has been derived previously for smooth asymptotically flat spacetimes [25]. Below, we find that this result extends to polyhomogeneous spacetimes [17]. Following Ref. [20], we extend the definition of BMS charges to subleading orders in a $1 / r$ expansion. Investigating these subleading BMS charges in the context of polyhomogeneous spacetimes is indeed the main aim of this paper. We will find that the results in the polyhomogeneous case are analogous to those for smooth spacetimes, albeit, the expressions are rather more complicated.

\section{A. BMS charge at $\mathcal{O}\left(r^{0}\right)$}

At leading order, we find

$$
\not \mathcal{I}_{0}=\delta\left(-2 s F_{0}\right)+\frac{s}{2} \partial_{u} B_{I J} \delta B^{I J} .
$$

Observe that at this leading order in the variation of the BMS charges (3.3), we do not encounter $\log r$ terms. This is a direct consequence of the finite shear condition (2.16), which implies that all leading order terms in the expansion (2.4) are independent of $z$.

As in the smooth case [25], the nonintegrability above is related to the existence of flux at infinity. In particular, the charge is integrable if and only if $\partial_{u} B_{I J}=0$, i.e., in the absence of Bondi news at null infinity [31]. The integrable part when integrated over the 2-sphere corresponds to leading-order BMS charges, which generalize the BondiSachs 4-momentum corresponding to $s$ an $\ell=0$ or 1 spherical harmonic.

\section{B. BMS charge at $\mathcal{O}\left(r^{-1}\right)$}

At the next order, we obtain

$$
\begin{aligned}
\not \mathcal{I}_{1}= & s \delta\left(-2 F_{1}-\left(1-\partial_{z}\right) D_{I} C_{1}^{I}+\frac{3}{16}(\square-2) B^{2}\right. \\
& \left.+D_{I} B^{I J} D^{K} B_{J K}-\frac{1}{4} D_{I} B_{J K} D^{I} B^{J K}\right) \\
& +\frac{1}{2} s\left(\partial_{u} B_{I J} \delta C^{I J}+\partial_{u} C_{I J} \delta B^{I J}\right) .
\end{aligned}
$$

If we assume the falloff condition on the matter fields $T_{m m}=o\left(r^{-3}\right)$, then from Eqs. (2.33) and (2.44), $\delta C_{I J}=$ $s \partial_{u} C_{I J}=0$, so the nonintegrable piece vanishes for all $s$. Assuming further that $T_{01}=o\left(r^{-4}\right)$ and $T_{0 m}=o\left(r^{-4}\right)$, then Eqs. (2.27) and (2.30) imply

$$
\delta \mathcal{I}_{1}=0 .
$$

Therefore, in this case $\mathcal{I}_{1}=0$ and there is no nontrivial charge. If, however, the falloff of $T_{01}$ is weaker, we have nonvanishing charges given by the coefficients of the polynomial in $z$ :

$$
\mathcal{Q}_{1}(z)=\int_{S} d \Omega\left(-\left.s T_{01}\right|_{r^{-4}}\right),
$$

provided that $T_{m m}=o\left(r^{-3}\right)$ and $T_{0 m}=o\left(r^{-4}\right)$. It can be shown by considering (2.47) that it is possible to have $T_{m m}=o\left(r^{-3}\right)$ and $T_{0 m}=o\left(r^{-4}\right)$ with $T_{01}$ nonvanishing at this order. The higher order charges depend only on $C_{I J}$. Since we have assumed $\partial_{u} C_{I J}=0$, such terms are trivially conserved. Therefore, the only interesting charge will be the one corresponding to the $z^{0}$ coefficient.

\section{BMS charge at $\mathcal{O}\left(r^{-2}\right)$}

Starting with weaker falloff conditions $T_{00}=o\left(r^{-5}\right)$, $T_{0 m}=o\left(r^{-4}\right)$ and $T_{m m}=o\left(r^{-3}\right)$, which imply Eqs. (2.25), (2.27) and (2.33), the variation of the BMS charge at the next order is 


$$
\begin{aligned}
\not \mathcal{I}_{2}= & s \delta\left(-2 F_{2}-\left(2-\partial_{z}\right) D_{I} C_{2}^{I}-3\left(C_{1}^{0}\right)^{I} D^{J} B_{I J}-\frac{3}{2} D^{J}\left(C_{1}^{0}\right)^{I} B_{I J}\right. \\
& -\frac{1}{4}\left(\int^{3}-2 \int+1\right) B_{I J} \square C^{I J}-\frac{1}{4}\left(\int-1\right) C_{I J} \square B^{I J}-\frac{1}{2}\left(\int+2 \int\right) B_{I J} C^{I J} \\
& -\frac{1}{2} \int^{3} D_{K} B_{I J} D^{K} C^{I J}+2 \int^{0} D_{I} B^{I J} D^{K} C_{J K}+\frac{1}{8} B^{2} D_{I} D_{J} B^{I J}-\frac{1}{32} B^{I J} D_{I} D_{J} B^{2} \\
& \left.-\frac{1}{8} B^{I J} D_{I} B_{K L} D_{J} B^{K L}+\frac{3}{16} D_{I} B^{I J} D_{J} B^{2}\right) \\
& +s\left(\frac{1}{2} \delta B^{I J} \partial_{u} D_{I J}+\frac{1}{2} \partial_{u} B^{I J} \delta D_{I J}-\frac{1}{16} \partial_{u} B^{2} \delta B^{2}+\frac{1}{8} F_{0} \delta B^{2}-\frac{1}{2} D^{J}\left(C_{1}^{0}\right)^{I} \delta B_{I J}\right. \\
& -\left(C_{1}^{0}\right)^{I} D^{J} \delta B_{I J}-\frac{1}{12}\left(2 \int^{3}-2 \int^{0}+3 \partial_{z}\right) \delta B_{I J} \square C^{I J}+\frac{1}{4}\left(2 \int^{3}+2-\partial_{z}\right) C_{I J} \square \delta B^{I J} \\
& -\frac{1}{6}\left(4 \int^{3}+2 \int^{0}-3+3 \partial_{z}\right) \delta B_{I J} C^{I J}+\frac{2}{3}\left(2 \int+\int^{3}\right) D_{K} \delta B_{I J} D^{K} C^{I J} \\
& +\frac{1}{2}\left(1-\partial_{z}\right) D_{I} \delta B^{I J} D^{K} C_{J K}+\frac{1}{16} D_{I} D_{J} B^{I J} \delta B^{2}+\frac{1}{32} D_{I} D_{J} B^{2} \delta B^{I J} \\
& \left.+\frac{1}{16} D_{I} B^{2} D_{J} \delta B^{I J}+\frac{1}{2} B^{I J} D^{K} B_{I K} D^{L} \delta B_{J L}+\frac{1}{8} \delta B^{I J} D_{I} B_{K L} D_{J} B^{K L}\right) .
\end{aligned}
$$

As ever, the above separation into the integrable and nonintegrable parts is not unique. The choice above has been made in order to obtain the simplest expressions possible. This will become most clear upon using further Einstein equations. If we further assume that $T_{0 m}=o\left(r^{-5}\right), T_{01}=o\left(r^{-5}\right)$ and $T_{m m}=o\left(r^{-4}\right)$, which imply Eqs. (2.28), (2.31) and (2.34), the above expression reduces to

$$
\begin{aligned}
\not \mathcal{I}_{2}= & s D_{I} D_{J} \delta\left(-D^{I J}+\frac{1}{16} B^{2} B^{I J}\right) \\
& +s\left(\frac{1}{2} \delta B^{I J} \partial_{u} D_{I J}+\frac{1}{2} \partial_{u} B^{I J} \delta D_{I J}-\frac{1}{16} \partial_{u} B^{2} \delta B^{2}+\frac{1}{8} F_{0} \delta B^{2}-\frac{1}{2} D^{J}\left(C_{1}^{0}\right)^{I} \delta B_{I J}\right. \\
& -\left(C_{1}^{0}\right)^{I} D^{J} \delta B_{I J}-\frac{1}{12}\left(2 \int-2 \int^{3}+3 \partial_{z}\right) \delta B_{I J} \square C^{I J}+\frac{1}{4}\left(2 \int^{3}+2-\partial_{z}\right) C_{I J} \square \delta B^{I J} \\
& -\frac{1}{6}\left(4 \int^{3}+2 \int-3+3 \partial_{z}\right) \delta B_{I J} C^{I J}+\frac{2}{3}\left(2^{3}+\int^{0}\right) D_{K} \delta B_{I J} D^{K} C^{I J} \\
& +\frac{1}{2}\left(1-\partial_{z}\right) D_{I} \delta B^{I J} D^{K} C_{J K}+\frac{1}{16} D_{I} D_{J} B^{I J} \delta B^{2}+\frac{1}{32} D_{I} D_{J} B^{2} \delta B^{I J} \\
& \left.+\frac{1}{16} D_{I} B^{2} D_{J} \delta B^{I J}+\frac{1}{2} B^{I J} D^{K} B_{I K} D^{L} \delta B_{J L}+\frac{1}{8} \delta B^{I J} D_{I} B_{K L} D_{J} B^{K L}\right)
\end{aligned}
$$

where for brevity, we have not directly substituted the expression for $\partial_{u} D_{I J}$. The integrable piece has $z$ degree $N_{D}>N_{C} \geq 0$. A nontrivial charge could appear as a coefficient of each $z$ power in the integrable piece. We first consider the highest order-the coefficient of $z^{N_{D}}$. The nonintegrable piece has maximum $z$ degree $N_{C}+1$ as can be seen from (2.38). If $N_{D}>N_{C}+1$ then each coefficient of $z^{n}$ for $n>N_{C}+1$ in the integrable piece gives a nontrivial charge. These are

$$
\mathcal{Q}_{2, n}^{\ell, m}=-\left.\frac{1}{16 \pi G} \int_{S} d \Omega Y_{\ell m} D_{I} D_{J} D^{I J}\right|_{\mathcal{O}\left(z^{n}\right)} \text { for } n>N_{C}+1
$$

where $Y_{\ell m}$ are spherical harmonics. However, inspecting Eq. (2.34), the Einstein equation for $\partial_{u} D_{I J}$, we notice that the right-hand side has $z$ degree $N_{C}+1$; hence the higher order terms in $D_{I J}$ do not evolve, i.e., they are constant in $u$. 
Therefore, the fact that the charges defined above are conserved is unsurprising.

The highest nontrivial order to consider is $\mathcal{O}\left(z^{N_{C}+1}\right)$. We must calculate the coefficient of $z^{N_{C}+1}$ in the nonintegrable piece and see what restrictions can be imposed in order to guarantee that this vanishes. The only terms in $\not \mathcal{I}_{2}^{\text {(non-int) }}$ that have $z$ dependence are those containing $C_{I J}$. Using Eqs. (2.34), (2.43) and (2.45), we obtain

$$
\begin{aligned}
\left.\not \mathcal{I}_{2}^{(\text {non-int })}\right|_{C_{I J} \text { terms }}= & D^{\langle I} D^{J\rangle} s\left(-\frac{1}{6}\left(4^{3}+2 \int^{0}+3-3 \partial_{z}\right) s C_{I J}\right. \\
& +\frac{1}{6}\left(2^{3}+\int^{0}\right) s \square C_{I J} \\
& -\left(2 \int^{3}+1\right) D^{K} C_{I J} D_{K} s \\
& +\frac{4}{3}\left(2 \int^{3}+\int^{0}\right) D^{K} C_{I K} D_{J} s \\
& \left.-\frac{1}{2}\left(2 \int^{3}+2-\partial_{z}\right) C_{I J} \square s\right) .
\end{aligned}
$$

Using the results of Appendix A, we find that the terms of $z$ degree $N_{C}+1$ in the expression above are of the form

$$
\begin{aligned}
\not \mathcal{I}_{2}^{\text {(non-int) }}= & \frac{1}{6} \int d z D^{\langle I} D^{J\rangle} s\left(s(\square-2) C_{I J}\right. \\
& \left.+8 D^{K} C_{K\langle I} D_{J\rangle} s\right)+\mathcal{O}\left(z^{N_{C}}\right) .
\end{aligned}
$$

For any given $s(x)$, we can choose a $C_{I J}(x)$ to make the expression in brackets an arbitrary symmetric traceless tensor. That is, for any traceless symmetric $X_{I J}(x)$ and $s\left(x^{I}\right)$, we can find a traceless symmetric solution $C_{I J}(x)$ to the second order partial differential equation:

$$
s(\square-2) C_{I J}+8 D^{K} C_{K\langle I} D_{J\rangle} s=X_{I J} .
$$

Since $C_{I J}(z, u, x)$ can be freely specified on a Cauchy surface, the expression in (3.13) can be made arbitrary on the surface and so (3.12) vanishes in general if and only if

$$
D_{\langle I} D_{J\rangle} s=0
$$

thus $s$ corresponds to an $\ell=0$ or 1 spherical harmonic (see Appendix C).
From (3.11), we observe that $\left.\not \mathcal{I}_{2}^{\text {(non-int) }}\right|_{C_{I J} \text { terms }}$ vanishes at all orders when $s$ obeys Eq. (3.14). Moreover, from Eq. (A13) in Appendix A, we have that for (3.11) to vanish at a given order, it must vanish at all higher orders, in particular the highest order. This means that $s$ must obey (3.14) for the coefficients of lower $z$ orders to be integrable. In conclusion, we deduce that at any order, (3.11) vanishes if and only if $s$ is an $\ell=0$ or 1 spherical harmonic.

Assuming Eq. (3.14), the nonintegrable part of Eq. (3.9) reduces to

$$
\begin{aligned}
\not \mathcal{I}_{2}^{(\text {non-int })}= & D^{I}\left(-s^{2}\left(C_{1}^{0}\right)^{J} \partial_{u} B_{I J}+\frac{1}{16} s^{2} D^{J} B^{2} \partial_{u} B_{I J}\right. \\
& \left.+\frac{1}{2} s^{2} B^{J K} D^{L} B_{K L} \partial_{u} B_{I J}\right)
\end{aligned}
$$

which is a total derivative and can, therefore, be ignored. Hence, at all orders in $z$ we obtain the (unintegrated) charges

$$
\mathcal{I}_{2}=s D_{I} D_{J}\left(-D^{I J}+\frac{1}{16} B^{2} B^{I J}\right)
$$

However, up to total derivatives, this is equal to

$$
\begin{aligned}
\mathcal{I}_{2} & =D_{I} D_{J} s\left(-D^{I J}+\frac{1}{16} B^{2} B^{I J}\right) \\
& =D_{\langle I} D_{J\rangle} s\left(-D^{I J}+\frac{1}{16} B^{2} B^{I J}\right) \\
& =0,
\end{aligned}
$$

where in the second line we have used the fact that $B_{I J}$ and $D_{I J}$ are trace-free and in the third line we have used Eq. (3.14). Therefore, the only nontrivial charges obtained at $\mathcal{O}\left(r^{-2}\right)$ are those given in Eq. (3.10).

\section{BMS charge at $\mathcal{O}\left(r^{-3}\right)$}

Starting with the previous falloff conditions $T_{00}=$ $o\left(r^{-5}\right), T_{0 m}=o\left(r^{-5}\right), T_{m m}=o\left(r^{-4}\right)$ and $T_{01}=o\left(r^{-5}\right)$, which imply Eqs. (2.25), (2.27), (2.28), (2.33) and (2.34), we find that, to leading orders in $z$, 


$$
\begin{aligned}
& \not \mathcal{I}_{3}=s \delta\left(-2 F_{3}+4 \beta_{2}+2 \square \beta_{2}-\left(3-\partial_{z}\right) D_{I} C_{3}^{I}-2 \int^{0}\left(C_{1}^{0}\right)^{I} D^{J} C_{I J}\right. \\
& +\frac{1}{2} \int^{1} B^{I J} \square D_{I J}+\frac{1}{2} D_{I J} \square B^{I J}+\frac{1}{2} D_{K} D_{I J} D^{K} B^{I J}+2 \int D_{I} B^{I J} D^{K} D_{J K} \\
& \left.-\frac{1}{2}\left(2 \int^{1}-1\right) B^{I J} D_{I J}-\frac{1}{4} \int^{0} D^{I}\left(B^{2} D^{J} C_{I J}\right)\right) \\
& +s\left(\frac{1}{2} \delta B^{I J} \partial_{u} E_{I J}+\frac{1}{2} \partial_{u} B^{I J} \delta E_{I J}-\frac{1}{12}\left(2 \int^{4}-2 \int^{1}-3+3 \partial_{z}\right) \delta B^{I J} \square D_{I J}\right. \\
& +\frac{1}{4}\left(2 \int^{4}+3-\partial_{z}\right) D_{I J} \square \delta B^{I J}+\frac{1}{2}\left(2-\partial_{z}\right) D_{K} D_{I J} D^{K} \delta B^{I J}+\frac{2}{3}\left(2 \int^{4}+\int^{1}\right) D_{I} \delta B^{I J} D^{K} D_{J K} \\
& -\frac{1}{6}\left(4 \int^{4}+2 \int^{1}-6+3 \partial_{z}\right) D_{I J} \delta B^{I J}+\int^{0}\left[\frac{5}{6} D^{I} \delta B^{2} D^{J} C_{I J}+\frac{3}{4} \delta B^{2} D^{I} D^{J} C_{I J}\right. \\
& -\frac{4}{3} B^{I J} \delta B_{I}{ }^{K} D_{J} D_{L} C_{K}^{L}-\frac{3}{2} B_{I}{ }^{K} \delta B^{I J} D_{J} D_{L} C_{K}{ }^{L}-2 B^{I J} D_{I} \delta B_{J K} D_{L} C^{K L} \\
& -\frac{3}{2} \delta B^{I J} D_{I} B_{J K} D_{L} C^{K L}-\frac{4}{3} \delta B_{I J} D_{K} B^{I K} D_{L} C^{J L}-\frac{11}{6} B_{I J} D_{K} \delta B^{I K} D_{L} C^{J L} \\
& \left.\left.-\frac{1}{6} \delta B^{I J} D^{K} B_{I J} D^{L} C_{K L}+\frac{1}{6} B^{I J} D^{K} \delta B_{I J} D^{L} C_{K L}\right]\right)+\mathcal{O}\left(z^{N_{C}}\right) \text {. }
\end{aligned}
$$

As before, it should be emphasized that the separation into integrable and nonintegrable pieces is not unique and the form above has been chosen to make the following expressions simpler.

Imposing a stronger falloff of the energy-momentum tensor, $T_{00}=o\left(r^{-6}\right), T_{01}=o\left(r^{-6}\right), T_{0 m}=o\left(r^{-6}\right)$ and $T_{m m}=o\left(r^{-5}\right)$ implies Eqs. (2.26), (2.32), (2.29) and
(2.35). Substituting these equations into the expressions above, in the integrable piece, terms will appear that depend only on $C_{I J}$. We drop such terms, since $\delta C_{I J}=0$ and one can add any arbitrary term depending only on $C_{I J}$ to the conserved charges. After applying some Schouten identities (see Appendix B), up to total derivatives

$$
\begin{aligned}
\not \mathcal{I}_{3}= & s \delta\left(-D_{I} D_{J} E^{I J}+\mathcal{O}\left(z^{N_{C}}\right)\right) \\
& +s\left(\frac{1}{2} \delta B^{I J} \partial_{u} E_{I J}+\frac{1}{2} \partial_{u} B^{I J} \delta E_{I J}-\frac{1}{12}\left(2 \int-2 \int-3+3 \partial_{z}\right) \delta B^{I J} \square D_{I J}\right. \\
& \left.+\frac{1}{4}\left(2 \int+3-\partial_{z}\right) D_{I J} \square \delta B^{I J}+\frac{1}{2}\left(2-\partial_{z}\right) D_{K} D_{I J} D^{K} \delta B^{I J}+\frac{2}{3}\left(2 \int+\int\right)^{1}\right) D_{I} \delta B^{I J} D^{K} D_{J K} \\
& -\frac{1}{6}\left(4 \int+2 \int-6+3 \partial_{z}\right) D_{I J} \delta B^{I J}+\int\left[\frac{5}{6} D^{I} \delta B^{2} D^{J} C_{I J}+\frac{3}{4} \delta B^{2} D^{I} D^{J} C_{I J}\right. \\
& -\frac{4}{3} B^{I J} \delta B_{I}{ }^{K} D_{J} D_{L} C_{K}{ }^{L}-\frac{3}{2} B_{I}{ }^{K} \delta B^{I J} D_{J} D_{L} C_{K}^{L}-2 B^{I J} D_{I} \delta B_{J K} D_{L} C^{K L} \\
& -\frac{3}{2} \delta B^{I J} D_{I} B_{J K} D_{L} C^{K L}-\frac{4}{3} \delta B_{I J} D_{K} B^{I K} D_{L} C^{J L}-\frac{11}{6} B_{I J} D_{K} \delta B^{I K} D_{L} C^{J L} \\
& \left.\left.-\frac{1}{6} \delta B^{I J} D^{K} B_{I J} D^{L} C_{K L}+\frac{1}{6} B^{I J} D^{K} \delta B_{I J} D^{L} C_{K L}\right]+\mathcal{O}\left(z^{N_{C}}\right)\right)
\end{aligned}
$$

where for brevity, we have not yet substituted in the expression for $\partial_{u} E_{I J}$. The integrable piece has $z$ degree $N_{E}$ where $N_{E} \geq N_{D}>N_{C}$. Using the appropriate Einstein equations and the metric variations shows that the nonintegrable piece has $z$ degree $N_{D}$. If $N_{E}>N_{D}$, terms $\mathcal{O}\left(z^{N_{D}+1}\right)$ or higher are integrable and we have charges

$$
\mathcal{Q}_{3, n}^{\ell, m}=-\left.\frac{1}{16 \pi G} \int_{S} d \Omega Y_{\ell m} D_{I} D_{J} E^{I J}\right|_{\mathcal{O}\left(z^{n}\right)} \quad \text { for } n>N_{D}
$$

As with charges (3.10) derived in Sec. III C, the existence of such conserved charges is unsurprising, since we observe 
from the Einstein equation (2.35) that terms of this degree in $E_{I J}$ are constant in $u$. The highest order at which the existence of a charge is not immediately obvious is at $\mathcal{O}\left(z^{N_{D}}\right)$. After applying the metric variations to (3.20) and tidying up the resulting expression using Schouten identities (see Appendix B), we find all $\partial_{u}$ terms at this order result in a total derivative and can hence be ignored. Recalling that $N_{C}<N_{D}$, it is possible that $N_{C}+1=N_{D}$ so the remaining terms in $\phi \mathcal{I}_{3}^{\text {(non-int) }}$ that can contribute to this order arise from $C_{I J}$ and $D_{I J}$ terms. We have

$$
\begin{aligned}
& \not \mathcal{I}_{3}^{\text {(non-int) }}=\left(\left.\not \varnothing \mathcal{I}_{3}^{\text {(non-int) }}\right|_{D \text { terms }}+\left.\not \mathcal{I}_{3}^{\text {(non-int })}\right|_{B C \text { terms }}\right) \\
& +\mathcal{O}\left(z^{N_{C}}\right) \text {, }
\end{aligned}
$$

where

$$
\begin{aligned}
\left.\not \mathcal{I}_{3}^{(\text {non-int })}\right|_{D \text { terms }}= & s\left(-\frac{1}{2}\left(\int^{1}+1-\partial_{z}\right) \square D^{I J} D_{I} D_{J} s+\frac{1}{2}\left(4 \int^{4}+2 \int^{1}-2+\partial_{z}\right) D^{I J} D_{I} D_{J} s\right. \\
& -\frac{1}{2}\left(2 \int^{4}+3-\partial_{z}\right) D^{I J} \square D_{I} D_{J} s-\left(2-\partial_{z}\right) D^{K} D^{I J} D_{K} D_{I} D_{J} s \\
& \left.-\frac{2}{3}\left(2 \int^{4}+\int^{1}\right) D_{I} D^{I J} D_{J} \square s-\frac{4}{3}\left(2 \int^{4}+\int^{1}\right) D_{I} D^{I J} D_{J} s\right)
\end{aligned}
$$

and

$$
\left.\not \mathcal{I}_{3}^{\text {(non-int) }}\right|_{B C \text { terms }}=\frac{1}{3} \int d z\left(B^{I J} D_{L} C^{K L} D_{K} D_{I} D_{J} s-\frac{1}{2} B^{I J} D_{K} C_{I}{ }^{K} D_{J} \square s-B^{I J} D_{K} C_{I}{ }^{K} D_{J} s\right)+\mathcal{O}\left(z^{N_{C}}\right) .
$$

There is no Einstein equation relating $D_{I J}$ and $C_{I J}$, so the contributions from the two terms above, namely $\left.\not \mathcal{I}_{3}^{\text {(non-int) }}\right|_{D \text { terms }}$ and $\left.\phi \mathcal{I}_{3}^{\text {(non-int) }}\right|_{B C \text { terms }}$, need to vanish independently in (3.20) in order for the charge to be integrable in general. We focus on $\left.\not \mathcal{I}_{3}^{(\text {non-int })}\right|_{D \text { terms }}$ to begin with. The $\mathcal{O}\left(z^{N_{D}}\right)$ coefficients can be calculated using (A2) in Appendix A. We find that

$$
\begin{aligned}
\left.\not \mathcal{I}_{3}^{(\text {non-int) }}\right|_{D \text { terms }}= & s\left(-\frac{5}{2} D^{I J} D_{I} D_{J} s-\frac{5}{4} D^{I J} \square D_{I} D_{J} s-2 D^{K} D^{I J} D_{K} D_{I} D_{J} s\right. \\
& \left.+D_{I} D^{I J} D_{J} \square s+2 D_{I} D^{I J} D_{J} s\right)\left.\right|_{\mathcal{O}\left(z^{N_{D}}\right)}+\mathcal{O}\left(z^{N_{D}-1}\right) .
\end{aligned}
$$

Now, in order to simplify this expression, we add to it an additional term,

$$
5 s\left(D^{I J} D_{I} D_{J} s+\frac{1}{4} D^{I J} D_{I} D_{J} \square s-\frac{1}{4} D^{I J} \square D_{I} D_{J} s\right),
$$

which vanishes upon use of the Ricci identity and the fact $D_{I J}$ is traceless; thus we have not changed the nonintegrable piece (3.25), which becomes

$$
\begin{aligned}
& \left.\not \mathcal{I}_{3}^{\text {(non-int) }}\right|_{D \text { terms }}=s\left(-\frac{5}{2} D^{I J} \square D_{I} D_{J} s+\frac{5}{4} D^{I J} D_{I} D_{J} \square s+\frac{5}{2} D^{I J} D_{I} D_{J} s\right. \\
& \left.-2 D^{K} D^{I J} D_{K} D_{I} D_{J} s+D_{I} D^{I J} D_{J} \square s+2 D_{I} D^{I J} D_{J} s\right)\left.\right|_{\mathcal{O}\left(z^{N_{D}}\right)}+\mathcal{O}\left(z^{N_{D}-1}\right) .
\end{aligned}
$$

Up to total derivatives, the first line in (3.27) can be written as

$$
\frac{5}{2} s D^{K} D^{I J} D_{K} D_{I} D_{J} s+\frac{5}{2} D^{I J} D^{K}{ }_{s} D_{K} D_{I} D_{J} s-\frac{5}{4} s D_{I} D^{I J} D_{J} \square s-\frac{5}{4} D^{I J} D_{I} s D_{J} \square s-\frac{5}{2} s D_{I} D^{I J} D_{J} s-\frac{5}{2} D^{I J} D_{I} s D_{J} s,
$$

so that 


$$
\begin{aligned}
\left.\not \mathcal{I}_{3}^{(\text {non-int })}\right|_{D \text { terms }}= & \left(\frac{1}{2} s D^{K} D^{I J} D_{K} D_{I} D_{J} s+\frac{5}{2} D^{I J} D^{K} s D_{K} D_{I} D_{J} s-\frac{1}{4} s D_{I} D^{I J} D_{J} \square s-\frac{5}{4} D^{I J} D_{I} s D_{J} \square s\right. \\
& \left.-\frac{1}{2} s D_{I} D^{I J} D_{J} s-\frac{5}{2} D^{I J} D_{I} s D_{J} s\right)\left.\right|_{\mathcal{O}\left(z^{N_{D}}\right)}+\mathcal{O}\left(z^{N_{D}-1}\right) \\
= & \left.\frac{1}{4}\left(s D^{K} D^{I J}+5 D^{I J} D^{K} s\right)\left(2 D_{K} D_{I} D_{J} s-\omega_{K I} D_{J} \square s-2 \omega_{I K} D_{J} s\right)\right|_{\mathcal{O}\left(z^{N} D\right)}+\mathcal{O}\left(z^{N_{D}-1}\right) .
\end{aligned}
$$

The factor $\left(s D_{K} D_{I J}+5 D_{I J} D_{K} s\right)$ is an arbitrary tensor that is symmetric and trace-free on its $I, J$ indices. Thus, for the $\mathcal{O}\left(z^{N_{D}}\right)$ term to vanish for general $D_{I J}$, it is necessary that the projection onto the traceless, symmetric part of the other factor vanishes, i.e.,

$$
2 D_{K} D_{\langle I} D_{J\rangle} s-\omega_{K\langle I} D_{J\rangle} \square s-2 \omega_{K\langle I} D_{J\rangle} s=0 .
$$

In Appendix C, we show that the above equation (3.30) is satisfied if and only if $s$ is a superposition of $\ell=0,1$ and 2 spherical harmonics. As with $\not \mathcal{I}_{2}$, there is little point considering $\ell=0$ and 1 modes since their contribution to the integrable piece is trivially zero; hence we now consider exclusively the case in which $s$ is an $\ell=2$ spherical harmonic, which means in particular that

$$
(\square+6) s=0 .
$$

Combining (3.31) with (3.30), we have

$$
\begin{aligned}
D_{K} D_{\langle I} D_{J\rangle} s-\frac{1}{3} \omega_{K\langle I} D_{J\rangle} \square s & =0 \quad \text { and } \\
D_{K} D_{\langle I} D_{J\rangle} s+2 \omega_{K\langle I} D_{J\rangle} s & =0 .
\end{aligned}
$$

Next, we consider the terms in $\left.\not \mathcal{I}_{3}^{\text {(non-int) }}\right|_{B C \text { terms }}$. Reorganizing these terms, Eq. (3.24) becomes

$$
\begin{aligned}
\left.\not \mathcal{I}_{3}^{\text {(non-int) }}\right|_{B C \text { terms }} & \\
= & \frac{1}{3} \int d z\left\{B^{I J} D_{L} C^{K L}\left(D_{K} D_{I} D_{J}-\frac{1}{3} \omega_{K I} D_{J} \square s\right)\right. \\
& \left.-\frac{1}{6} B^{I J} D_{K} C_{I}{ }^{K} D_{J}(\square+6) s\right\}+\mathcal{O}\left(z^{N_{C}}\right) .
\end{aligned}
$$

Given that $B_{I J}$ is trace-free and symmetric, we can use Eqs. (3.32) and (3.31) to conclude that

$$
\left.\not \mathcal{I}_{3}^{\text {(non-int) }}\right|_{B C \text { terms }}=0
$$

Therefore, even in the extreme case $N_{D}=N_{C}+1$, the $\mathcal{O}\left(z^{N_{D}}\right)$ term still vanishes for an $\ell=2$ spherical harmonic. The fact that the nonintegrable charges vanish at $z$ degree $N_{C}+1$ means that we have five conserved charges

$$
\mathcal{Q}_{m}=-\left.\frac{1}{16 \pi G} \int_{S} d \Omega Y_{2 m} D_{I} D_{J} E^{I J}\right|_{\mathcal{O}\left(z^{N D}\right)} \text { for } m=0, \pm 1, \pm 2
$$

We shall show below in Sec. V that these charges correspond to half of the set of Newman-Penrose charges that exist in such polyhomogeneous spacetimes [18].

We now consider the nonintegrable piece at lower orders. Using the result in (A13), for the nonintegrable piece to vanish at lower orders for general $D_{I J}$, it is necessary that it vanishes at the highest order for general $D_{I J}$ and so $s$ must be an $\ell=2$ spherical harmonic. In this case, applying Eq. (A8) to the expression in (3.23), the contribution from $D_{I J}$ terms at $\mathcal{O}\left(z^{N_{D}-1}\right)$ is

$$
\begin{aligned}
\left.\not \mathcal{I}_{3}^{\text {(non-int) }}\right|_{D \text { terms }}= & N_{D} s\left(\square D^{I J} D_{I} D_{J} s-\frac{5}{8} D^{I J} D_{I} D_{J} s+\frac{9}{16} D^{I J} \square D_{I} D_{J} s\right. \\
& \left.+D^{K} D^{I J} D_{K} D_{I} D_{J} s+\frac{3}{4} D_{I} D^{I J} D_{J} \square s+\frac{3}{2} D_{I} D^{I J} D_{J} s\right)\left.\right|_{\mathcal{O}\left(z^{N^{-1}}\right)}+\mathcal{O}\left(z^{N_{D}-2}\right) .
\end{aligned}
$$

Up to total derivatives, this becomes 


$$
\begin{aligned}
\left.\not \mathcal{I}_{3}^{(\text {non-int })}\right|_{D \text { terms }}= & N_{D} D^{I J}\left(\frac{9}{16} s \square D_{I} D_{J} s-\frac{3}{4} s D_{I} D_{J} \square s+D^{K} D_{K} D_{I} D_{J} s-\frac{3}{4} D_{I} \square s D_{J} s\right. \\
& \left.+\square s D_{I} D_{J} s-\frac{17}{8} s D_{I} D_{J} s-\frac{3}{2} D_{I} s D_{J} s\right)\left.\right|_{\mathcal{O}\left(z^{N^{-1}}\right)}+\mathcal{O}\left(z^{N_{D}-2}\right)
\end{aligned}
$$

Then using Eqs. (3.31) and (3.32), this reduces to

$$
\begin{aligned}
& \left.\not \mathcal{I}_{3}^{\text {(non-int) }}\right|_{D \text { terms }} \\
& =\left.N_{D} D^{I J}\left(-\frac{19}{4} s D_{I} D_{J} s+D_{I} s D_{J} s\right)\right|_{\mathcal{O}\left(z^{N-1}\right)} \\
& \quad+\mathcal{O}\left(z^{N_{D}-2}\right)
\end{aligned}
$$

which for general $D_{I J}$ and $s$ an $\ell=2$ spherical harmonic is not zero. Any further restriction on $s$ will make the integrable piece vanish. There is no need to check $\left.\not \mathcal{I}_{3}^{\text {(non-int) }}\right|_{B C \text { terms }}$ at this order since there is no equation linking $D_{I J}$ to $C_{I J}$ and $B_{I J}$ that could result in a cancellation in the nonintegrable piece. We deduce that $\left.\not \mathcal{I}_{3}^{\text {(non-int) }}\right|_{D \text { terms }}$ is nonvanishing at this order and hence there are no charges at this order, nor subsequent orders as implied by (A13).

In summary, the complete set of conserved charges obtained at $\mathcal{O}\left(r^{-3}\right)$ is given by (3.21) and (3.35).

\section{DUAL BMS CHARGES}

We now turn to the tower of dual charges defined in Ref. [21], given by the expression

$$
\begin{aligned}
\not \tilde{\mathcal{Q}}_{\xi}[\delta g, g] & =\frac{1}{8 \pi G} \int_{S} \tilde{H}[\xi, g, \delta g] \\
& =\frac{1}{8 \pi G} \int_{S} d \Omega \frac{\tilde{H}_{\theta \phi}[\xi, g, \delta g]}{\sin \theta}
\end{aligned}
$$

where we have used the form of the background metric of interest (2.2) in the second equality with the 2-form $\tilde{H}$ given by

$$
\tilde{H}=\frac{1}{4} \delta g_{b c}\left(\nabla_{a} \xi^{c}+\nabla^{c} \xi_{a}\right) d x^{a} \wedge d x^{b}
$$

The dual BMS charges can be derived from first principles from the Palatini-Holst action [27,28]. We will consider a $1 / r$ expansion of the variation of the dual BMS charge:

$$
\begin{aligned}
\not \tilde{\mathcal{Q}}_{\xi}[\delta g, g]= & \frac{1}{16 \pi G} \int_{S} d \Omega\left\{\phi \tilde{\mathcal{I}}_{0}+\frac{\phi \tilde{\mathcal{I}}_{1}(z)}{r}+\frac{\not \tilde{\mathcal{I}}_{2}(z)}{r^{2}}\right. \\
& \left.+\frac{\phi \tilde{\mathcal{I}}_{3}(z)}{r^{3}}+o\left(r^{-3}\right)\right\} .
\end{aligned}
$$

The calculations will be analogous to those in Sec. III with similar results being obtained, as with the smooth case [21].

Following Ref. [26], it will be useful to define the twist of a symmetric tensor $X_{I J}$ :

$$
\tilde{X}^{I J}=X_{K}{ }^{(I} \epsilon^{J) K}, \quad \epsilon_{I J}=\left(\begin{array}{cc}
0 & 1 \\
-1 & 0
\end{array}\right) \sin \theta .
$$

Note, if $X^{I J}$ is trace-free, we can drop the symmetrization in the definition (4.4). Additionally, it is helpful to note if $X$ and $Y$ are both symmetric trace-free tensors, then

$$
X_{I K} \tilde{Y}^{J K}=-\tilde{X}_{I K} Y^{J K}
$$

Furthermore, if either one of the symmetric tensors $X$ or $Y$ is trace-free, then

$$
X_{I J} \tilde{Y}^{I J}=-\tilde{X}_{I J} Y^{I J}
$$

With the above definitions in mind, Eq. (4.1) can be written as

$$
\phi \tilde{\mathcal{Q}}_{\xi}[\delta g, g]=\frac{1}{16 \pi G} \int_{S} d \Omega \epsilon^{I J} \tilde{H}_{I J}[\xi, g, \delta g]
$$

We now proceed as before to substitute the metric expansions (2.4) and the expression for $\xi$ given in Eq. (2.40).

\section{A. Dual charge at $\mathcal{O}\left(\boldsymbol{r}^{0}\right)$}

At leading order, we find

$$
\not \tilde{\mathcal{I}}_{0}=\delta\left(-s D_{I} D_{J} \tilde{B}^{I J}\right)+\frac{1}{2} s \partial_{u} B_{I J} \delta \tilde{B}^{I J}
$$

As with (3.4), we have an integrable piece that is in general nonzero and a nonintegrable piece that vanishes if and only if $\partial_{u} B_{I J}=0$, i.e., in the absence of Bondi news. In this case, we have an infinite set of conserved charges,

$$
\tilde{\mathcal{Q}}_{0}^{\ell, m}=-\frac{1}{16 \pi G} \int_{S} d \Omega Y_{\ell m} D_{I} D_{J} \tilde{B}^{I J}
$$

which are to be viewed as the generalization of the NUT charge [26]; see also Ref. [32]. 


\section{B. Dual charge at $\mathcal{O}\left(r^{-1}\right)$}

At the next order, we find

$$
\not \tilde{\mathcal{I}}_{1}=\delta\left(-s D_{I} D_{J} \tilde{C}^{I J}\right)+\frac{1}{2} s\left(\partial_{u} C_{I J} \delta \tilde{B}^{I J}-\delta C_{I J} \partial_{u} \tilde{B}^{I J}\right) .
$$

If we assume $T_{m m}=o\left(r^{-3}\right)$, which implies Eq. (2.33), then using Eq. (2.44), we get $\not \tilde{\mathcal{I}}_{1}=0$ so

$$
\tilde{\mathcal{I}}_{1}=0 \text {. }
$$

This is analogous to the $\mathcal{O}\left(r^{-1}\right)$ term in Sec. III B, where we found that the charge is zero if strong enough falloff conditions on the energy-momentum tensor are assumed.

\section{Dual charge at $\mathcal{O}\left(\boldsymbol{r}^{-2}\right)$}

At the next order, we find

$$
\begin{aligned}
\not \tilde{\mathcal{I}}_{2}= & s D_{I} D_{J} \delta\left(-\tilde{D}^{I J}+\frac{1}{16} B^{2} \tilde{B}^{I J}\right) \\
& +s\left(\frac{1}{2}\left(\partial_{u} D_{I J} \delta \tilde{B}^{I J}-\delta D_{I J} \partial_{u} \tilde{B}^{I J}\right)-\frac{1}{16} B_{I J}\left(\partial_{u} B^{2} \delta \tilde{B}^{I J}-\delta B^{2} \partial_{u} \tilde{B}^{I J}\right)\right. \\
& +\frac{1}{2} \partial_{u} C_{I J} \delta \tilde{C}^{I J}+D_{I}\left(C_{1 J} \delta \tilde{B}^{I J}\right)-\frac{1}{2}\left(\int^{3}+1\right) C_{I J} \square \delta \tilde{B}^{I J}+\frac{1}{4} \partial_{z}\left(\square\left(C_{I J} \delta \tilde{B}^{I J}\right)\right) \\
& \left.+\int^{3} C_{I J} \delta \tilde{B}^{I J}-\frac{1}{2} D_{K} C_{I J} D^{K} \delta \tilde{B}^{I J}-\int^{3} D^{K} C_{J K} D_{I} \delta \tilde{B}^{I J}-\frac{1}{16} D_{I}\left(D_{J} B^{2} \delta \tilde{B}^{I J}\right)-\frac{1}{2} D_{I}\left(B_{J K} D_{L} B^{K L} \delta \tilde{B}^{I J}\right)\right) .
\end{aligned}
$$

The integrable piece has $z$ degree $N_{D}$, whereas from (2.38) we deduce that the nonintegrable piece has $z$ degree at most $N_{C}+1 \leq N_{D}$. In the case $N_{D}>N_{C}+1$, we have charges

$\tilde{\mathcal{Q}}_{2, n}^{\ell, m}=-\left.\frac{1}{16 \pi G} \int_{S} d \Omega Y_{\ell m} D_{I} D_{J} \tilde{D}^{I J}\right|_{\mathcal{O}\left(z^{n}\right)}$ for $n>N_{C}+1$.

This is analogous to the result in Sec. III C, where we found a set of charges (3.10). As with those charges, the existence of these conserved charges is unsurprising when we consider the Einstein equation (2.34). Thus, as in Sec. III C, the highest nontrivial order is $\mathcal{O}\left(z^{N_{C}+1}\right)$, which we consider next. All terms with $z$ dependence arise from the presence of $C_{I J}$, so we start by considering such terms. Assume that $T_{0 m}=o\left(r^{-4}\right)$, which implies Eq. (2.27). Rewriting

$$
\begin{aligned}
& s\left(\partial_{u} D_{I J} \delta \tilde{B}^{I J}-\delta D_{I J} \partial_{u} \tilde{B}^{I J}\right) \\
& =-\left(\delta D_{I J}-s \partial_{u} D_{I J}\right) \delta \tilde{B}^{I J}+\delta D_{I J}\left(\delta \tilde{B}^{I J}-s \partial_{u} \tilde{B}^{I J}\right)
\end{aligned}
$$

and assuming $T_{m m}=o\left(r^{-4}\right)$, i.e., Eqs. (2.34), and (2.45) in the first term in (4.14) and (2.43) in the second term, we get

$$
\begin{aligned}
\left.\not \tilde{\mathcal{I}}_{2}{ }^{\text {(non-int) }}\right|_{C \text { terms }} & \\
= & \left.D^{\langle I} D^{J\rangle} s \delta \tilde{D}_{I J}\right|_{C \text { terms }} \\
= & \frac{1}{6} \int d z D^{\langle I} D^{J\rangle} s\left(s(\square-2) \tilde{C}_{I J}+8 D^{K} \tilde{C}_{K\langle I} D_{J\rangle} s\right) \\
& +\mathcal{O}\left(z^{N_{C}}\right),
\end{aligned}
$$

where we have used Eqs. (2.34) and (2.45). This is the same expression as was obtained in (3.12), except that the tensor field $C_{I J}$ has been twisted. Since $\tilde{C}^{I J}$ is also an arbitrary symmetric, traceless tensor, we again deduce that the highest order term in $\not \tilde{\mathcal{I}}_{2}{ }^{\text {(non-int) }}$ is zero if and only if $D_{\langle I} D_{J\rangle} s=0$, i.e., if $s$ is an $\ell=0$ or 1 spherical harmonic. Assuming this to be the case, as we can see from Eq. (4.15), this implies that the $C_{I J}$ terms vanish at all orders in $z$. Furthermore, $D_{\langle I} D_{J\rangle} s=0$ implies that (2.43) reduces to $\delta B_{I J}=s \partial_{u} B_{I J}$. Thus, the nonintegrable term in Eq. (4.12) reduces to

$$
\begin{aligned}
\not \tilde{\mathcal{I}}_{2} \text { (non-int) }= & D^{I}\left(s^{2}\left(C_{1}^{0}\right)^{J} \partial_{u} \tilde{B}_{I J}-\frac{1}{2} s^{2} B^{J K} D^{L} B_{K L} \partial_{u} \tilde{B}_{I J}\right. \\
& \left.-\frac{1}{16} s^{2} D^{J} B^{2} \partial_{u} \tilde{B}_{I J}\right) \\
& +\frac{1}{16} s D^{\langle I} D^{J\rangle} s\left(B^{2} \partial_{u} \tilde{B}_{I J}+2 B^{K L} \partial_{u} \tilde{B}_{K L} B_{I J}\right),
\end{aligned}
$$

where the first line is a total derivative and so can be ignored and the second line vanishes through our choice of $s$. As was the case in Sec. III C, the highest order term in the nonintegrable piece vanishes if and only if the nonintegrable piece vanishes at all orders, with the reverse argument following from (A13) in Appendix A.

With $s$ an $\ell=0$ or 1 spherical harmonic, up to total derivatives, the integrable piece becomes

$$
\begin{aligned}
\delta \tilde{\mathcal{I}}_{2} & =D_{I} D_{J} s \delta\left(-\tilde{D}^{I J}+\frac{1}{16} B^{2} \tilde{B}^{I J}\right) \\
& =D_{\langle I} D_{J\rangle} s \delta\left(-\tilde{D}^{I J}+\frac{1}{16} B^{2} \tilde{B}^{I J}\right) \\
& =0
\end{aligned}
$$

So as before, there is no nontrivial charge at this order. 


\section{Dual charge at $\mathcal{O}\left(r^{-3}\right)$}

At the next order, we find that

$$
\begin{aligned}
\not \tilde{\mathcal{I}}_{3}= & -s D_{I} D_{J} \delta \tilde{E}^{I J} \\
& +s\left(\frac{1}{2}\left(\partial_{u} E_{I J} \delta \tilde{B}^{I J}-\delta E_{I J} \partial_{u} \tilde{B}^{I J}\right)+\frac{1}{4} D_{I}\left(3 C_{1}^{K} B_{J K} \delta \tilde{B}^{I J}-C_{1}^{K} B^{I J} \delta \tilde{B}_{J K}\right)\right. \\
& +D_{I}\left(C_{2 J} \delta \tilde{B}^{I J}\right)-\frac{1}{4}\left(1-\partial_{z}\right) \delta \tilde{B}^{I J} \square D_{I J}-\frac{1}{4}\left(2 \int^{4}+3-\partial_{z}\right) D_{I J} \square \delta \tilde{B}^{I J}+\int^{4} \delta \tilde{B}^{I J} D_{I J} \\
& \left.-\frac{1}{2}\left(2-\partial_{z}\right) D_{K} D_{I J} D^{K} \delta \tilde{B}^{I J}-\int^{4} D_{K} D^{J K} D^{I} \delta \tilde{B}_{I J}\right)+\mathcal{O}\left(z^{N_{C}}\right),
\end{aligned}
$$

where we have used Eqs. (2.33) and (2.44) to drop all terms involving only $C_{I J}$. The integrable piece has $z$ degree $N_{E}$, whereas (2.38) implies that the nonintegrable piece has $z$ degree at most $N_{D} \leq N_{E}$. We therefore have a set of conserved charges:

$\tilde{\mathcal{Q}}_{3, n}^{\ell, m}=-\left.\frac{1}{16 \pi G} \int_{S} d \Omega Y_{\ell m} D_{I} D_{J} \tilde{E}^{I J}\right|_{\mathcal{O}\left(z^{n}\right)} \quad$ for $n>N_{D}$.

Once again, this is unsurprising, when we consider the form of the Einstein equation (2.35).

Next, we consider the highest order term in $\not \tilde{\mathcal{I}}_{3}$ (non-int) and see if it is possible to make this zero in general for a particular choice of $s\left(x^{I}\right)$. The highest order term is $\mathcal{O}\left(z^{N_{D}}\right)$, but in the extreme case where $N_{D}=N_{C}+1$, it is essential that $\mathcal{O}\left(z^{N_{C}+1}\right)$ terms also vanish. We further assume $T_{0 m}=o\left(r^{-5}\right)$ and $T_{m m}=o\left(r^{-5}\right)$. Rewriting

$$
\begin{aligned}
& s\left(\partial_{u} E_{I J} \delta \tilde{B}^{I J}-\delta E_{I J} \partial_{u} \tilde{B}^{I J}\right) \\
& \quad=-\left(\delta E_{I J}-s \partial_{u} E_{I J}\right) \delta \tilde{B}^{I J}+\delta E_{I J}\left(\delta \tilde{B}^{I J}-s \partial_{u} \tilde{B}^{I J}\right)
\end{aligned}
$$

in (4.18) and using Eqs. (2.35) and (2.46) in the first set of terms and (2.43) on the second set of terms, as well as Eqs. (2.27) and (2.28) gives

$$
\begin{aligned}
\not \tilde{\mathcal{I}}_{3}{ }^{\text {(non-int) }}= & D^{I} D^{J} s \delta \tilde{E}_{I J}+\mathcal{O}\left(z^{N_{C}}\right) \\
= & D^{I} D^{J} s\left(\left.\tilde{X}_{\langle I J\rangle}\right|_{\mathcal{O}\left(z^{N_{D}}\right)}+\left.\tilde{Y}_{\langle I J\rangle}\right|_{\mathcal{O}\left(z^{N_{C}+1}\right)}\right)+\mathcal{O}\left(z^{N_{C}}\right) \\
= & D^{\langle I} D^{J\rangle} s\left(\left.\tilde{X}_{\langle I J\rangle}\right|_{\mathcal{O}\left(z^{N_{D}}\right)}+\left.\tilde{Y}_{\langle I J\rangle}\right|_{\mathcal{O}\left(z^{N+1}\right)}\right) \\
& +\mathcal{O}\left(z^{N_{C}}\right),
\end{aligned}
$$

where

$$
\begin{aligned}
X_{I J}= & \frac{1}{3}\left(2 \int^{4}+\int^{1}\right) s D_{I} D^{K} D_{J K}-\frac{1}{2}\left(2-\partial_{z}\right) s D_{I J} \\
& -\frac{1}{2}\left(2 \int^{4}+3-\partial_{z}\right) D_{I J} \square s \\
& +\frac{1}{3}\left(2 \int^{4}+4 \int-3\right) D^{K} D_{I K} D_{J} s \\
& -\left(2 \int^{4}+1\right) D_{I} D_{J K} D^{K} s
\end{aligned}
$$

and

$$
\begin{aligned}
Y_{I J}= & \frac{1}{6} \int d z\left(s B^{K L} D_{I} D_{J} C_{K L}\right. \\
& -s B_{I J} D_{K} D_{L} C^{K L}-s B^{K L} D_{K} D_{L} C_{I J} \\
& -2 s D^{K} B_{J K} D^{L} C_{L I}+2 B_{J K} D_{L} C^{K L} D_{I} s \\
& \left.-2 B_{I J} D_{L} C^{K L} D_{K} s\right) .
\end{aligned}
$$

Note that only the symmetric traceless part of $\tilde{X}_{I J}$ and $\tilde{Y}_{I J}$, and therefore $X_{I J}$ and $Y_{I J}$, need be considered.

The contributions from the $X_{I J}$ terms and $Y_{I J}$ terms need to vanish independently in (4.21), as there is no Einstein equation that relates $D_{I J}$ and $C_{I J}$. First, we focus on $X_{I J}$. Use of the Ricci identity and the Schouten identity (B4) allows us to rewrite $X_{I J}$ (up to the symmetric, trace-free part) as

$$
\begin{aligned}
X_{I J}= & \frac{1}{3}\left(2 \int^{4}+\int^{1}\right) s D^{K} D_{I} D_{J K}-\left(2 \int^{4}+1\right) D_{I} D_{J K} D^{K} s \\
& -\left(2 \int^{4}+3-\partial_{z}\right) D_{J K} D^{K} D_{I} s \\
& +\frac{1}{3}\left(2 \int^{4}+4 \int^{1}-3\right) D^{K} D_{J K} D_{I} s \\
& -\frac{1}{6}\left(8 \int^{4}+4 \int+6-3 \partial_{z}\right) s D_{I J}
\end{aligned}
$$

Then using Eq. (A2), we find that the highest order term is 


$$
\begin{aligned}
\left.X_{I J}\right|_{\mathcal{O}\left(z^{N_{D}}\right)} & =\left.\left(-\frac{1}{2} s D^{K} D_{I} D_{J K}-\frac{1}{2} D_{I} D_{J K} D^{K} s-\frac{5}{2} D_{J K} D^{K} D_{I} s-\frac{5}{2} D^{K} D_{J K} D_{I} s\right)\right|_{\mathcal{O}\left(z^{N}\right)} \\
& =\left.D^{K}\left(-\frac{1}{2} s D_{I} D_{J K}-\frac{5}{2} D_{J K} D_{I} s\right)\right|_{\mathcal{O}\left(z^{N_{D}}\right)} \cdot
\end{aligned}
$$

So in (4.21), the contribution from $X_{I J}$ is

$$
\begin{aligned}
\left.\not \tilde{\mathcal{I}}_{3}{ }^{\text {(non-int) }}\right|_{D \text { terms }} & =-\left.\frac{1}{2} D^{\langle I} D^{J\rangle} s D^{K}\left(s D_{I} \tilde{D}_{J K}+5 \tilde{D}_{J K} D_{I} s\right)\right|_{\mathcal{O}\left(z^{N_{D}}\right)}+\mathcal{O}\left(z^{N_{D}-1}\right) \\
& =\left.\frac{1}{2} D^{K} D^{\langle I} D^{J\rangle} s\left(s D_{I} \tilde{D}_{J K}+5 \tilde{D}_{J K} D_{I} s\right)\right|_{\mathcal{O}\left(z^{N_{D}}\right)}+\mathcal{O}\left(z^{N_{D}-1}\right)
\end{aligned}
$$

up to total derivatives. The expression $s D_{I} \tilde{D}_{J K}+5 \tilde{D}_{J K} D_{I} s$ is an arbitrary tensor that is symmetric and trace-free on its $J, K$ indices. The contribution to $\not \tilde{\mathcal{I}}_{3}{ }^{\text {(non-int) }}$ from $D_{I J}$ terms therefore vanishes if and only if the projection of $D_{K} D_{\langle I} D_{J\rangle} s$ onto the symmetric trace-free part in the $J K$ indices is zero. It is shown in Sec. 5.4 of Ref. [21] that this condition is satisfied if and only if $s$ is an $\ell=0,1$ or 2 spherical harmonic. As in Sec. III D, the contribution of the $\ell=0,1$ modes vanishes in the integrable piece and so can be ignored. Henceforth, we assume that $s$ is an $\ell=2$ spherical harmonic satisfying Eqs. (3.31) and (3.32).

Turning our attention to the $Y_{I J}$ contributions in (4.21), the expression in (4.23) can be rewritten as

$$
\begin{aligned}
Y_{I J}= & -\frac{1}{3} \int d z D^{K}\left(s B_{J K} D^{L} C_{I L}\right) \\
& +\frac{1}{6} s \int d z\left(B^{K L} D_{I} D_{J} C_{K L}-B_{I J} D_{K} D_{L} C^{K L}\right. \\
& \left.-B^{K L} D_{K} D_{L} C_{I J}+2 B_{J K} D^{K} D^{L} C_{I L}\right) \\
& +\frac{1}{3} \int d z\left(B_{J K} D_{L} C^{K L} D_{I} s-B_{I J} D_{L} C^{K L} D_{K} s\right. \\
& \left.+B_{J K} D^{L} C_{I L} D^{K} s\right) .
\end{aligned}
$$

Since $B_{I J}$ and $C_{I J}$ are symmetric and traceless, using Schouten identities (B5) and (B6), the second and third lines have zero trace-free symmetric parts and hence can be ignored. The contribution of the $Y_{I J}$ terms to $\not \tilde{\mathcal{I}}_{3}$ (non-int) is therefore simply

$$
\begin{aligned}
& \left.\not \tilde{\mathcal{I}}_{3}{ }^{\text {(non-int) }}\right|_{C \text { terms }} \\
& =-\left.\frac{1}{3} D^{\langle I} D^{J\rangle} s \int d z D^{K}\left(s B_{J K} \nabla^{L} C_{I L}\right)\right|_{\mathcal{O}\left(z^{N} C^{+1}\right)}+\mathcal{O}\left(z^{N_{C}}\right) \\
& =\left.\frac{1}{3} s D^{K} D^{\langle I} D^{J\rangle} s \int d z B_{J K} \nabla^{L} C_{I L}\right|_{\mathcal{O}\left(z^{N} C^{+1}\right)}+\mathcal{O}\left(z^{N_{C}}\right) \text {, }
\end{aligned}
$$

up to total derivatives. Since $B_{J K}$ is symmetric and traceless, $D^{K} D^{\langle I} D^{J\rangle} s$ is projected onto the symmetric trace-free part on its $J K$ indices, which vanishes given that $s$ is an $\ell=2$ spherical harmonic. We conclude that for $s$ an $\ell=2$ spherical harmonic, the $\mathcal{O}\left(z^{N_{D}}\right)$ terms in $\phi \tilde{\mathcal{I}}_{3}{ }^{\text {(non-int) }}$ vanish even in the extreme case $N_{C}+1=N_{D}$.

In summary, we have a set of conserved nontrivial charges,

$\tilde{\mathcal{Q}}_{m}=-\left.\frac{1}{16 \pi G} \int_{S} d \Omega Y_{2 m} D_{I} D_{J} \tilde{E}^{I J}\right|_{\mathcal{O}\left(z^{N_{D}}\right)}$ for $m=0, \pm 1, \pm 2$

We now turn our attention to the lower order terms. Again, from (A13), if $\not \tilde{\mathcal{I}}_{3}{ }^{\text {(non-int) }}$ cannot be made to vanish at a particular order, then it cannot vanish at any lower orders when the tensor fields being considered are arbitrary. We will consider $\mathcal{O}\left(z^{N_{D}-1}\right)$ and show that the $D_{I J}$ terms cannot be made to vanish at this order, confirming that there are no further charges at lower orders. Using (A8), we find that there are two independent contributions at $\mathcal{O}\left(z^{N_{D}-1}\right)$ that must vanish independently. The first has the same form as the highest order $D_{I J}$ terms so vanishes if and only if $s$ is an $\ell=2$ spherical harmonic. Using (A2), the remaining contribution from the second term is

$$
\begin{aligned}
\left.\not \tilde{\mathcal{I}}_{3}{ }^{\text {(non-int) }}\right|_{D \text { terms }} & N_{D} D^{\langle I} D^{J\rangle} s\left(-\frac{3}{8} s D^{K} D_{I} \tilde{D}_{J K}+\frac{1}{8} D_{I} \tilde{D}_{J K} D^{K} s+\frac{5}{4} s \tilde{D}_{I J}\right. \\
& \left.+\frac{9}{8} \tilde{D}_{I K} D_{J} D^{K} s-\frac{11}{8} D^{K} \tilde{D}_{I K} D_{J} s\right)\left.\right|_{\mathcal{O}\left(z^{N-1}\right)}
\end{aligned}
$$

Reorganizing the terms above gives 


$$
\begin{aligned}
\left.\not \tilde{\mathcal{I}}_{3}{ }^{(\text {non-int })}\right|_{D \text { terms }}= & N_{D} D^{\langle I} D^{J\rangle} s\left(\frac{1}{8}\left(s D^{K} D_{I} \tilde{D}_{J K}+D_{I} \tilde{D}_{J K} D^{K} s\right)-\frac{1}{2} s D^{K} D_{I} \tilde{D}_{J K}\right. \\
& \left.+\frac{5}{4} s \tilde{D}_{I J}-\frac{11}{8}\left(\tilde{D}_{I K} D_{J} D^{K} s+D^{K} \tilde{D}_{I K} D_{J} s\right)+\frac{5}{2} \tilde{D}_{I K} D_{J} D^{K} s\right)\left.\right|_{\mathcal{O}\left(z^{N^{-1}}\right)} \\
= & \left.N_{D} D^{\langle I} D^{J\rangle}\left(-\frac{1}{2} s D^{K} D_{I} \tilde{D}_{J K}+\frac{5}{4} s \tilde{D}_{I J}+\frac{5}{2} \tilde{D}_{I K} D_{J} D^{K} s\right)\right|_{\mathcal{O}\left(z^{N-1}\right)},
\end{aligned}
$$

where we have integrated by parts and used the fact that $s$ is an $\ell=2$ spherical harmonic. Applying Schouten identities, integrating by parts and applying the equations for an $\ell=2$ spherical harmonic, we obtain

$$
\begin{aligned}
& \left.\not \tilde{\mathcal{I}}_{3}{ }^{\text {(non-int) }}\right|_{D \text { terms }} \\
& =\left.N_{D} \tilde{D}^{I J}\left(-\frac{19}{4} s D_{I} D_{J} s+D_{I} s D_{J} s\right)\right|_{\mathcal{O}\left(z^{N^{N}-1}\right)} \\
& \quad+\mathcal{O}\left(z^{N_{D}-2}\right)
\end{aligned}
$$

The obstruction that prevents an integrable charge existing at this order is exactly the twist of the obstruction in $\left.\not \mathcal{I}_{3}^{\text {(non-int) }}\right|_{D \text { terms }}$ in (3.38).

In conclusion, the set of conserved charges that can be found by considering $\not \tilde{\mathcal{I}}_{3}$ are given by (4.19) and (4.29).

\section{RELATING CHARGES TO THE NEWMAN PENROSE FORMALISM}

In this section, we relate the charges obtained here to quantities in the Newman-Penrose formalism [3,18,22]. At $\mathcal{O}\left(r^{-3}\right)$, we will see that the BMS charge and dual charge together form a generalization of the Newman-Penrose charges for polyhomogeneous spacetimes with finite shear.

The Newman-Penrose formalism begins with a complex null frame $\{\ell, n, m, \bar{m}\}$, which we choose to be that given in (2.10). Newman-Penrose scalars are then constructed by contracting tensors into null frame components. One such set of complex scalars are the Weyl scalars, given in Eq. (2.8), which parametrize the ten degrees of freedom of the Weyl tensor. We reproduce these definitions here for convenience:

$$
\begin{array}{ll}
\Psi_{0}=\ell^{a} m^{b} \ell^{c} m^{d} C_{a b c d}, & \Psi_{1}=\ell^{a} n^{b} \ell^{c} m^{d} C_{a b c d}, \\
\Psi_{2}=\ell^{a} m^{b} \bar{m}^{c} n^{d} C_{a b c d}, & \Psi_{3}=\ell^{a} n^{b} \bar{m}^{c} n^{d} C_{a b c d}, \\
\Psi_{4}=n^{a} \bar{m}^{b} n^{c} \bar{m}^{d} C_{a b c d} . &
\end{array}
$$

The Riemann tensor is constructed from the Weyl tensor and the Ricci tensor and the ten degrees of freedom of the Ricci tensor, which is constrained by the Einstein equation, are given by three complex and four real scalars. The relevant quantities here are

$$
\Lambda=-\frac{1}{24} R, \quad \Phi_{11}=-\frac{1}{4} \ell^{a} n^{b} R_{a b}-\frac{1}{4} m^{a} \bar{m}^{b} R_{a b},
$$

both of which are real. Similarly, the connection coefficients may be written in terms of 12 complex scalars. For our purposes, we will only be interested in one such spin coefficient that parametrizes the shear of the null congruence generated by the vector field $\ell$,

$$
\sigma=-m^{a} m^{b} \nabla_{b} \ell_{a}
$$

All such quantities can be calculated from the metric (2.2) and (2.4). We assume that the energy-momentum tensor falls off as $T_{00}=o\left(r^{-5}\right), T_{0 m}=o\left(r^{-4}\right)$ and $T_{01}=$ $o\left(r^{-3}\right)$. Then one can show that the Weyl scalars fall off as [19]

$$
\begin{aligned}
\Psi_{0}= & \Psi_{0}^{4}\left[N_{C}\right] r^{-4}+\Psi_{0}^{5}\left[N_{D}\right] r^{-5}+\Psi_{0}^{6}\left[N_{E}\right] r^{-6} \\
& +\mathcal{O}\left(r^{-7} \log ^{N_{1}} r\right), \\
\Psi_{1}= & \Psi_{1}^{4}\left[N_{C}+1\right] r^{-4}+\mathcal{O}\left(r^{-5} \log ^{N_{2}} r\right), \\
\Psi_{2}= & \Psi_{2}^{3}[0] r^{-3}+\Psi_{2}^{4}\left[N_{C}+1\right] r^{-4}+\mathcal{O}\left(r^{-5} \log ^{N_{3}} r\right), \\
\Psi_{3}= & \mathcal{O}\left(r^{-2}\right), \quad \Psi_{4}=\mathcal{O}\left(r^{-1}\right),
\end{aligned}
$$

where the quantities in square brackets in each expression refer to the $z$ degree of each polynomial. The exact values of $N_{1}, N_{2}$ and $N_{3}$ are not important for what we are concerned with. The leading order shear term is independent of $z$,

$$
\sigma=\sigma^{0}[0] \frac{1}{r^{2}}
$$

which follows from condition (2.16). Furthermore, ${ }^{5}$

$$
\begin{gathered}
\Lambda=\Lambda_{4}\left[N_{4}\right] r^{-4}+\mathcal{O}\left(r^{-5} \log ^{N_{5}} r\right), \\
\Phi_{11}=\Phi_{11}^{4}\left[N_{4}+1\right]+\mathcal{O}\left(r^{-5} \log ^{N_{6}} r\right),
\end{gathered}
$$

where the exact values of $N_{5}$ and $N_{6}$ are unimportant.

Finally, we define the differential operators $ð$ and $\bar{\partial}$, which act on a scalar $\eta$ of spin $n$ as follows $[3,33]$ :

\footnotetext{
${ }^{5}$ If one assumes $T_{01}=o\left(r^{-4}\right)$, then $N_{4}=N_{C}+1$.
} 


$$
\begin{gathered}
ð \eta=-\frac{(1+i)}{2} \sin ^{n} \theta\left(\partial_{\theta}-\frac{1}{\sin \theta} \partial_{\phi}\right)\left(\frac{\eta}{\sin ^{n} \theta}\right), \\
\bar{\partial} \eta=-\frac{(1-i)}{2} \frac{1}{\sin ^{n} \theta}\left(\partial_{\theta}+\frac{1}{\sin \theta} \partial_{\phi}\right)\left(\sin ^{n} \theta \eta\right) .
\end{gathered}
$$

A Weyl scalar $\Psi_{n}$ has spin $2-n$, while the shear $\sigma$ has spin 2. Complex conjugation changes the sign of the spin.

\section{A. Charges at $\mathcal{O}\left(\boldsymbol{r}^{0}\right)$}

At leading order, we obtained the BMS charges and the dual charges in Secs. III A and IVA,

$$
\begin{aligned}
& \mathcal{Q}_{0}^{(\mathrm{int})}=\frac{1}{16 \pi G} \int_{S} d \Omega\left(-2 s F_{0}\right), \\
& \tilde{\mathcal{Q}}_{0}^{\text {(int) }}=\frac{1}{16 \pi G} \int_{S} d \Omega\left(-s D_{I} D_{J} \tilde{B}^{I J}\right) .
\end{aligned}
$$

Recall that the leading order charges are integrable if and only if $\partial_{u} B_{I J}=0$. We define a complex quantity,

$$
\mathcal{Q}_{0}=\mathcal{Q}_{0}^{(\mathrm{int})}-i \tilde{\mathcal{Q}}_{0}^{(\mathrm{int})} .
$$

In terms of Newman-Penrose quantities,

$$
\mathcal{Q}_{0}=-\frac{1}{4 \pi G} \int_{S} d \Omega s\left(\Psi_{2}^{3}+\sigma^{0} \partial_{u} \bar{\sigma}^{0}\right)
$$

which is conserved if and only if $\partial_{u} \sigma^{0}=0$. This condition is equivalent to $\partial_{u} B_{I J}=0$; the integrability condition encountered in Secs. III A and IVA.

\section{B. Charges at $\mathcal{O}\left(\boldsymbol{r}^{-1}\right)$}

In Secs. III B and IV B, assuming that $T_{m m}=o\left(r^{-3}\right)$ and $T_{0 m}=o\left(r^{-4}\right)$, we obtained the following set of integrable charges at the next order $^{6}$ :

$$
\begin{aligned}
\mathcal{Q}_{1}^{(\text {int })}(z)= & \frac{1}{16 \pi G} \int_{S} d \Omega s\left(-2 F_{1}-\left(1-\partial_{z}\right) D_{I} C_{1}^{I}\right. \\
& +\frac{3}{16}(\square-2) B^{2}+D_{I} B^{I J} D^{K} B_{J K} \\
& \left.-\frac{1}{4} D_{I} B_{J K} D^{I} B^{J K}\right), \\
\tilde{\mathcal{Q}}_{1}^{(\text {int })}(z)= & \frac{1}{16 \pi G} \int_{S} d \Omega\left(-s D_{I} D_{J} \tilde{C}^{I J}\right) .
\end{aligned}
$$

Note that the coefficient of each power of $z$ is an independent charge. Letting

$$
\mathcal{Q}_{1}=\mathcal{Q}_{1}^{(\mathrm{int})}-i \tilde{\mathcal{Q}}_{1}^{(\mathrm{int})},
$$

\footnotetext{
${ }^{6}$ The dual charge here is trivially conserved and vanished in Sec. IV C by virtue of the fact that $\delta C_{I J}=0$.
}

it can be shown that

$$
\mathcal{Q}_{1}=\frac{1}{4 \pi G} \int_{S} d \Omega s\left(\left(\int^{2}-\int^{1}\right) \bar{\delta}^{2} \Psi_{0}^{4}-\int^{1}\left(\Phi_{11}^{4}+3 \Lambda_{4}\right)\right)
$$

The first term is trivially conserved since $T_{m m}=o\left(r^{-3}\right)$ implies $\partial_{u} \Psi_{0}^{4}=0$. Assuming $T_{01}=o\left(r^{-4}\right)$ makes the second term zero. The second term is real and gives the nontrivial conserved charges (3.7) in Sec. III B when the falloff of the energy-momentum tensor is not too strong.

\section{Charges at $\mathcal{O}\left(\boldsymbol{r}^{-2}\right)$}

At the next order, in Secs. III C and IV C, we obtained the charges

$$
\mathcal{Q}_{2}^{(\mathrm{int})}(z)=\frac{1}{16 \pi G} \int_{S} d \Omega s D_{I} D_{J}\left(-D^{I J}+\frac{1}{16} B^{2} B^{I J}\right),
$$

$\tilde{\mathcal{Q}}_{2}^{\text {(int) }}(z)=\frac{1}{16 \pi G} \int_{S} d \Omega s D_{I} D_{J}\left(-\tilde{D}^{I J}+\frac{1}{16} B^{2} \tilde{B}^{I J}\right)$

and showed that the associated nonintegrable terms vanished for $s$ an $\ell=0,1$ spherical harmonic. It can be shown that

$$
\begin{aligned}
\bar{\jmath}^{2} \Psi_{0}^{5}= & D_{I} D_{J}\left(-\frac{1}{4}\left(\partial_{z}^{2}-5 \partial_{z}+6\right)\left(D^{I J}-i \tilde{D}^{I J}\right)\right. \\
& \left.+\frac{3}{32} B^{2}\left(B^{I J}-i \tilde{B}^{I J}\right)\right)
\end{aligned}
$$

and

$$
\left[-\frac{1}{4}\left(\partial_{z}^{2}-5 \partial_{z}+6\right)\right]^{-1}=-4\left(\int^{3}-\int^{2}\right)
$$

as an operator equation. Thus,

$$
\begin{aligned}
& -4\left(\int^{3}-\int^{2}\right) \bar{\partial}^{2} \Psi_{0}^{5} \\
& =D_{I} D_{J}\left(\left(D^{I J}-i \tilde{D}^{I J}\right)-\frac{1}{16} B^{2}\left(B^{I J}-i \tilde{B}^{I J}\right)\right),
\end{aligned}
$$

where we have used Eq. (2.16), i.e., that $B_{I J}$ is $z$ independent. Defining

$$
\mathcal{Q}_{2}(z)=\mathcal{Q}_{2}^{(\mathrm{int})}(z)-i \tilde{\mathcal{Q}}_{2}^{(\mathrm{int})}(z),
$$

it can be shown that the charges obtained in Secs. III C and IV C at $\mathcal{O}\left(z^{N_{C}+1}\right)$ and lower can be written in terms of Newman-Penrose quantities as 


$$
\mathcal{Q}_{2}(z)=\frac{1}{4 \pi G} \int_{S} d \Omega s\left(\int^{3}-\int^{2}\right) \bar{\partial}^{2} \Psi_{0}^{5},
$$

where each coefficient of a $z$ power in $\mathcal{Q}_{2}(z)$ is an independent conserved charge. Integrating by parts, the differential operators can be moved onto $s$ confirming that this is zero for $s$ an $\ell=0,1$ spherical harmonic, since $\overline{\mathrm{d}}^{2} Y_{\ell m}=0$ for $\ell=0,1$.

\section{Charges at $\mathcal{O}\left(\boldsymbol{r}^{-3}\right)$}

Finally, and most interestingly, in Secs. III D and IV D, we obtained the charges

$$
\begin{aligned}
& \mathcal{Q}_{3}^{\text {(int) }}=\left.\frac{1}{16 \pi G} \int_{S} d \Omega s\left(-D_{I} D_{J} E^{I J}\right)\right|_{\mathcal{O}\left(z^{N_{D}}\right)}, \\
& \tilde{\mathcal{Q}}_{3}^{\text {(int) }}=\left.\frac{1}{16 \pi G} \int_{S} d \Omega s\left(-D_{I} D_{J} \tilde{E}^{I J}\right)\right|_{\mathcal{O}\left(z^{N_{D}}\right)},
\end{aligned}
$$

and showed that the associated nonintegrable pieces vanished for $s$ an $\ell=0,1$ or 2 spherical harmonic. It can be shown that

$$
\begin{aligned}
\bar{\partial}^{2} \Psi_{0}^{6}= & -\frac{1}{4}\left(\partial_{z}^{2}-7 \partial_{z}+12\right) D_{I} D_{J}\left(E^{I J}-i \tilde{E}^{I J}\right) \\
& +\mathcal{O}\left(z^{N_{C}}\right)
\end{aligned}
$$

and

$$
\left[-\frac{1}{4}\left(\partial_{z}^{2}-7 \partial_{z}+12\right)\right]^{-1}=-4\left(\int-\int^{3}\right)
$$

as an operator equation. Thus,

$-4\left(\int-\int^{4}\right) \bar{\jmath}^{2} \Psi_{0}^{6}=D_{I} D_{J}\left(E^{I J}-i \tilde{E}^{I J}\right)+\mathcal{O}\left(z^{N_{C}}\right)$.

Defining

$$
\mathcal{Q}_{3}=\mathcal{Q}_{3}^{(\mathrm{int})}-i \tilde{\mathcal{Q}}_{3}^{(\mathrm{int})},
$$

it can be shown that the charges obtained in Secs. III D and IV D can be written in terms of Newman-Penrose quantities as

$$
\mathcal{Q}_{3}=\left.\frac{1}{4 \pi G} \int_{S} d \Omega s\left(\int^{4}-\int^{3}\right) \bar{\jmath}^{2} \Psi_{0}^{6}\right|_{\mathcal{O}\left(z^{N_{D}}\right)} .
$$

Recalling that, furthermore, we have another set of lessinteresting conserved charges (3.21) and (4.19), we readily deduce that the expression

$$
\frac{1}{16 \pi G} \int_{S} d \Omega s\left[-\left.D_{I} D_{J}\left(E^{I J}-i \tilde{E}^{I J}\right)\right|_{\mathcal{O}\left(z^{N}\right)}\right]
$$

is a conserved charge for $N>N_{D}$ and any $s$, including, in particular, when $s$ is an $\ell=0,1$ or 2 spherical harmonic. The $\partial_{z}$ terms in (5.24) evaluated at $\mathcal{O}\left(z^{N_{D}}\right)$ carry contributions only from charges (5.29) and hence it is possible to produce a more simple expression for the charge built out of Newman-Penrose quantities given by

$\mathcal{Q}^{\mathcal{V K}}{ }_{3}=\left.\frac{1}{48 \pi G} \int_{S} d \Omega Y_{2, m} \bar{\partial}^{2} \Psi_{0}^{6}\right|_{\mathcal{O}\left(z^{N_{D}}\right)} \quad$ for $m=0, \pm 1, \pm 2$.

Integrating by parts, we obtain the generalization found in Ref. [18] for the Newman-Penrose charges of polyhomogeneous spacetimes with finite shear. For a smooth spacetime, $N_{D}=0$; hence the above expression reduces to the original Newman-Penrose charges [22].

\section{ACKNOWLEDGMENTS}

We would like to thank Hadi Godazgar, Chris Pope and Juan Valiente Kroon for discussions. M. G. is supported by a Royal Society University Research Fellowship. G. L. is supported by a Royal Society Enhancement Award.

\section{APPENDIX A: POLYNOMIALS IN $z=\log r$}

In this Appendix, we collect some useful properties of polynomials in $z$. For $\lambda \in \mathbb{R} \backslash\{0\}$ and $n \in \mathbb{N}_{\geq 0}$

$$
\begin{aligned}
\int z^{n} & \equiv e^{\lambda z} \int d z e^{-\lambda z} z^{n} \\
& =e^{\lambda z}\left(-\frac{1}{\lambda} e^{-\lambda z} z^{n}+\frac{n}{\lambda} \int d z e^{-\lambda z} z^{n-1}\right) \\
& =e^{\lambda z}\left(-\frac{1}{\lambda} e^{-\lambda z} z^{n}-\frac{n}{\lambda^{2}} e^{-\lambda z} z^{n-1}\right)+\mathcal{O}\left(z^{n-2}\right) \\
& =-\frac{1}{\lambda} z^{n}-\frac{n}{\lambda^{2}} z^{n-1}+\mathcal{O}\left(z^{n-2}\right) .
\end{aligned}
$$

Let $p(z)=p_{n} z^{n}+p_{n-1} z^{n-1}+\mathcal{O}\left(z^{n-2}\right)$ be a polynomial in $z$, then using (A1), we have by linearity of $\int^{\lambda}$

$\int^{\lambda} p(z)=-\frac{1}{\lambda} p_{n} z^{n}-\left(\frac{n}{\lambda^{2}} p_{n}+\frac{1}{\lambda} p_{n-1}\right) z^{n-1}+\mathcal{O}\left(z^{n-2}\right)$.

Also,

$$
\partial_{z} p(z)=n p_{n} z^{n-1}+\mathcal{O}\left(z^{n-2}\right) .
$$

In particular, note that for $c$ independent of $z$,

$$
\int^{\lambda} c=-\frac{1}{\lambda} c .
$$

If we apply a generic linear operator $\mathcal{O}$ formed of $\partial_{z}$, 1 and $\int^{\lambda}$ with $\lambda \neq 0$ to any $p(z)=p_{n} z^{n}+p_{n-1} z^{n-1}$, we get 
a new polynomial $\tilde{p}(z)=\mathcal{O} p(z)$ of the same degree, which can be expressed in the form

$\tilde{p}(z)=A p_{n} z^{n}+\left(A p_{n-1}+n B p_{n}\right) z^{n-1}+\mathcal{O}\left(z^{n-2}\right)$

with $A$ and $B n$-independent constants depending on the choice of $\mathcal{O}$.

Let $X$ be some tensor of interest, depending on $\left(u, r, x^{I}\right)$ where the $r$ dependence is such that $X$ can be written as a polynomial in $z=\log r$ with coefficients depending on $\left(u, x^{I}\right)$, so $X=\sum_{i=0}^{n} X_{i} z^{i}$ where $X_{i}$ are tensors of the same rank as $X$ and independent of $z$. Taking angular derivatives of such an expression, for example $\square X$, gives an expression of the form $F\left(D_{I}\right)[X]=\sum_{i=0}^{n} Y\left(X_{i}\right) z^{i}$, where at each order the same function $Y$ appears. Suppose we have an expression $P_{X}(z)$ involving the $\log r$ operators above, $X$ and its derivatives, where $X$ only appears linearly. In general, we can decompose such as object as follows:

$$
P_{X}(z)=\sum_{a} \mathcal{O}_{a} F_{a}\left(D_{I}\right)[X]
$$

where $F_{a}\left(D_{I}\right)[X]=\sum_{i=0}^{n} Y_{a}\left(X_{i}\right) z^{i}$ for some $Y_{a}$. Then by Eq. (A5), we can write

$$
\begin{aligned}
\mathcal{O}_{a} F_{a}\left(D_{I}\right)[X]= & A_{a} Y_{a}\left(X_{n}\right) z^{n} \\
& +\left(A_{a} Y_{a}\left(X_{n-1}\right)+n B_{a} Y_{a}\left(X_{n}\right)\right) z^{n-1} \\
& +\mathcal{O}\left(z^{n-2}\right),
\end{aligned}
$$

which implies that Eq. (A6) reduces to

$$
\begin{aligned}
P_{X}(z)= & \sum_{a} \mathcal{O}_{a} F_{a}\left(D_{I}\right)[X] \\
= & \sum_{a} A_{a} Y_{a}\left(X_{n}\right) z^{n} \\
& +\left(\sum_{a} A_{a} Y_{a}\left(X_{n-1}\right)+n \sum_{a} B_{a} Y_{a}\left(X_{n}\right)\right) z^{n-1} \\
& +\mathcal{O}\left(z^{n-2}\right) .
\end{aligned}
$$

Now, we consider making this expression vanish at various orders for general $X$. This expression vanishes at the highest order $z^{n}$, if and only if

$$
\sum_{a} A_{a} Y_{a}\left(X_{n}\right)=0
$$

Note that because we assume $X$ to be some arbitrary tensor, the above equation must hold as an operator equation and should not be viewed an equation for $X_{n}$. At the next order $z^{n-1}$, there are two terms that need to vanish independently since one depends only on $X_{n}$ and the other depends only on $X_{n-1}$, which are not necessarily related. So the $z^{n-1}$ coefficient vanishes if and only if

$$
\sum_{a} A_{a} Y_{a}\left(X_{n-1}\right)=0 \quad \text { and } \quad \sum_{a} B_{a} Y_{a}\left(X_{n}\right)=0
$$

Since both $X_{n}$ and $X_{n-1}$ are arbitrary, these conditions show that if $P_{X}(z)$ vanishes at the highest order, checking that it vanishes at the second highest order only requires one to check that $\sum_{a} B_{a} Y_{a}\left(X_{n}\right)=0$ for some arbitrary $X_{n}$. Furthermore,

$$
\left.P_{X}(z)\right|_{z^{n-1}}=\left.0 \Rightarrow P_{X}(z)\right|_{z^{n}}=0 .
$$

This argument can be extended to all orders, where at each order a new condition arises, but the previous conditions must still be met. We deduce for general $X$

$$
\left.P_{X}(z)\right|_{z^{i-1}}=\left.0 \Rightarrow P_{X}(z)\right|_{z^{i}}=0
$$

for $i=1, \ldots, n$. In particular, considering the contrapositive,

$$
\left.P_{X}(z)\right|_{z^{i}} \neq\left. 0 \Rightarrow P_{X}(z)\right|_{z^{i-1}} \neq 0 \quad \forall 1 \leq i \leq n,
$$

i.e., in order for the expression $P_{X}(z)$ to vanish at a particular order for general $X$, it needs to vanish at all higher orders for general $X$.

\section{APPENDIX B: IDENTITIES FOR TENSORS ON THE 2-SPHERE}

Schouten identities have been used extensively in this paper to simplify expressions. For a traceless, symmetric tensor $X_{I J}$, the Schouten identity implies that [20]

$$
\omega_{I J} X_{K L}+\omega_{K L} X_{I J}-\omega_{I L} X_{J K}-\omega_{J K} X_{I L}=0 .
$$

This equation can be used as the starting point for deriving further useful identities. In addition to those identities listed in Appendix B of Ref. [20], in this Appendix, we list a few other important examples. For $X_{I J}$ and $Y_{I J}$ arbitrary symmetric, traceless tensors and $s$ some arbitrary scalar,

$$
\begin{gathered}
X_{\langle I}{ }^{K} Y_{J\rangle K}=0, \\
D_{\langle I} D^{K} X_{J\rangle K}=\frac{1}{2} \square X_{I J}-X_{I J}, \\
X_{K\langle I} D_{J\rangle} D^{K} s=\frac{1}{2} X_{I J} \square s, \\
X_{I J} D_{L} Y^{K L} D_{K} s-X_{\langle I| K} D_{L} Y^{K L} D_{|J\rangle} s-X_{\langle I| K} D^{L} Y_{|I\rangle L} D^{K} s=0,
\end{gathered}
$$

$$
\begin{aligned}
& Y_{I J} D_{K} D_{L} X^{K L}+Y^{K L} D_{K} D_{L} X_{I J}-Y^{K L} D_{\langle I} D_{J\rangle} X_{K L} \\
& \quad-2 Y_{\langle I| K} D^{K} D^{L} X_{|J\rangle L}=0 .
\end{aligned}
$$

We briefly explain in turn how the above identities are obtained from Eq. (B1). Contracting (B1) with symmetric 
traceless tensor $Y^{K L}$ gives (B2). Next, we apply the derivative operator $D^{L} D^{K}$ on Eq. (B1). This gives

$$
\omega_{I J} D^{K} D^{L} X_{K L}+\square X_{I J}-D_{I} D^{K} X_{J K}-D^{K} D_{J} X_{I K}=0 .
$$

Making use of the Ricci identity and the form of the Riemann tensor

$$
R_{J K L}^{I}=\delta_{K}^{I} \omega_{J L}-\delta_{L}^{I} \omega_{J K}
$$

for the standard 2-sphere metric $\omega_{I J}$, we get

$$
\begin{gathered}
\omega_{I J} D^{K} D^{L} X_{K L}+\square X_{I J}-D_{I} D^{K} X_{J K} \\
-D_{J} D^{K} X_{I K}-2 X_{I J}=0,
\end{gathered}
$$

which is equivalent to (B3). This is an important identity that is used frequently in this paper. Similarly, contracting (B1) with $D_{K} D_{L} s$ gives (B4).

Contracting Eq. (B1) with $D_{M} Y^{K M} D^{L} s$ and taking the symmetric trace-free part of the resulting equation gives Eq. (B5).

Finally, we apply $Y^{K M} D^{L} D_{M}$ as an operator on Eq. (B1) to obtain

$$
\begin{aligned}
& \omega_{I J} Y^{K M} D^{L} D_{M} X_{K L}+Y^{K L} D_{K} D_{L} X_{I J}-Y^{K L} D_{I} D_{L} X_{J K} \\
& \quad-Y_{J K} D^{L} D^{K} X_{I L}=0 .
\end{aligned}
$$

Now, relabeling $I \rightarrow M$ in Eq. (B1) and acting with $Y^{M L} D_{I} D^{K}$ gives

$Y_{J L} D_{I} D_{K} X^{K L}+Y^{K L} D_{I} D_{L} X_{J K}-Y^{K L} D_{I} D_{J} X_{K L}=0$.

Using Eq. (B11) to substitute for the $Y^{K L} D_{I} D_{L} X_{J K}$ term in Eq. (B10) gives

$$
\begin{gathered}
\omega_{I J} Y^{K M} D^{L} D_{M} X_{K L}+Y^{K L} D_{K} D_{L} X_{I J}+Y_{J L} D_{I} D_{K} X^{K L} \\
-Y^{K L} D_{I} D_{J} X_{K L}-Y_{J K} D^{L} D^{K} X_{I L}=0 .
\end{gathered}
$$

Next, replacing $X \rightarrow Y$ in Eq. (B1) and acting with $D^{L} D_{M} X^{K M}$ gives

$$
\begin{aligned}
& \omega_{I J} Y^{K M} D_{M} D^{L} X_{K L}+Y_{I J} D_{K} D_{L} X^{K L} \\
& \quad-Y_{J L} D_{I} D_{K} X^{K L}-Y_{I K} D^{K} D^{L} X_{J L}=0,
\end{aligned}
$$

which we use to replace the $Y_{J L} D_{I} D_{K} X^{K L}$ term in Eq. (B12), resulting in

$$
\begin{aligned}
& \omega_{I J} Y^{K M} D^{L} D_{M} X_{K L}+Y^{K L} D_{K} D_{L} X_{I J}+\omega_{I J} Y^{K M} D_{M} D^{L} X_{K L} \\
& \quad+Y_{I J} D_{K} D_{L} X^{K L}-Y_{I K} D^{K} D^{L} X_{J L}-Y^{K L} D_{I} D_{J} X_{K L} \\
& \quad-Y_{J K} D^{L} D^{K} X_{I L}=0 .
\end{aligned}
$$

We use the Ricci identity to exchange the $D_{K}$ and $D_{L}$ derivatives in the last term. This results in an additional term of the form $X_{I K} Y_{J}{ }^{K}$. Now, taking the symmetric tracefree part of this equation and using Eq. (B2) yields identity (B6).

\section{APPENDIX C: $\ell=0, \ell=1$ AND $\ell=2$ SPHERICAL HARMONICS}

In this Appendix, we list useful properties of $\ell \leq 2$ spherical harmonics. This Appendix has a large overlap with Appendix C of Ref. [20]. However, given the importance of these results in this paper, for completeness, we reproduce the relevant equations here. A regular function $\psi\left(x^{I}\right)$ on the sphere can be written in terms of an expansion

$$
\psi\left(x^{I}\right)=\sum_{\ell=0}^{\infty} \sum_{m=-\ell}^{l} \psi^{\ell m} Y_{\ell m}\left(x^{I}\right)
$$

with $\psi^{\ell m}$ constants on the sphere and the spherical harmonics $Y_{\ell m}\left(x^{I}\right)$ with $\ell \geq 0$ and $|m| \leq \ell$ obeying

$$
\square Y_{\ell m}=-\ell(\ell+1) Y_{\ell m} .
$$

Consider the equation

$$
D_{\langle I} D_{J\rangle} \psi=0 .
$$

Let $T_{I J}=D_{\langle I} D_{J\rangle} \psi$. Integrating by parts and using the Ricci identity, it can be shown

$$
\int_{S} d \Omega\left|T_{I J}\right|^{2}=\frac{1}{2} \int_{S} d \Omega \psi \square(\square+2) \psi .
$$

If $\psi\left(x^{I}\right)$ is regular, we can assume the expansion (C1). Plugging this into (C4) and using the orthogonality relations for spherical harmonics $\int_{S} d \Omega Y_{\ell m} Y_{\ell^{\prime} m^{\prime}}=$ $\delta_{\ell \ell^{\prime}} \delta_{m m^{\prime}}$, we find

$$
\begin{aligned}
& \int_{S} d \Omega\left|T_{I J}\right|^{2} \\
&=\frac{1}{2} \int_{S} d \Omega \sum_{\ell=0}^{\infty} \sum_{\ell^{\prime}=0}^{\infty} \sum_{m=-\ell}^{\ell} \sum_{m^{\prime}=-\ell^{\prime}}^{\ell^{\prime}}(\ell-1) \ell(\ell+1)(\ell+2) \\
& \times \psi^{\ell m} \psi^{\ell^{\prime} m^{\prime}} Y_{\ell m} Y_{\ell^{\prime} m^{\prime}} \\
&= \frac{1}{2} \sum_{\ell=0}^{\infty} \sum_{m=-\ell}^{\ell}(\ell-1) \ell(\ell+1)(\ell+2)\left|\psi^{\ell m}\right|^{2} .
\end{aligned}
$$

Notice that each term in the summation on the rhs is nonnegative. Therefore, for the rhs to vanish, all terms must individually vanish, which implies that the rhs vanishes if and only if $\psi^{\ell m}=0$ for all $\ell>1$, i.e., $\psi\left(x^{I}\right)$ is a linear combination of $\ell=0$ and $\ell=1$ modes. We conclude, 
then, that Eq. (C3) holds if and only if $\psi\left(x^{I}\right)$ is a linear combination of $\ell=0$ and $\ell=1$ modes.

Consider now Eq. (3.30), which is equivalent to

$$
\begin{aligned}
& T_{I J K}=0, \\
& T_{I J K}=2 D_{K} D_{\langle I} D_{J\rangle} \psi-\omega_{K\langle I} D_{J\rangle} \square \psi-2 \omega_{K\langle I} D_{J\rangle} \psi .
\end{aligned}
$$

For a function $\psi\left(x^{I}\right)$ that is regular on the sphere, integration by parts can be used to show that

$$
\int_{S} d \Omega\left|T_{I J K}\right|^{2}=-\int_{S} d \Omega \psi \square(\square+2)(\square+6) \psi .
$$

Inserting expansion $(\mathrm{C} 1)$ into the above equation yields

$$
\begin{aligned}
\int_{S} d \Omega\left|T_{I J K}\right|^{2}= & \sum_{\ell=0}^{\infty} \sum_{\ell=-m}^{m}(\ell-2)(\ell-1) \ell(\ell+1) \\
& \times(\ell+2)(\ell+3)\left|\psi^{\ell m}\right|^{2} .
\end{aligned}
$$

Using the same argument as above, we deduce that $T_{I J K}=$ 0 if and only if $\psi\left(x^{I}\right)$ is a linear combination of $\ell=0,1$ and 2 spherical harmonics.
[1] H. Bondi, M. G. J. Van der Burg, and A. W. K. Metzner, Gravitational waves in general relativity, VII. Waves from axi-symmetric isolated system, Proc. R. Soc. A 269, 21 (1962).

[2] R. K. Sachs, Gravitational waves in general relativity VIII. Waves in asymptotically flat space-time, Proc. R. Soc. A 270, 103 (1962).

[3] E. Newman and R. Penrose, An approach to gravitational radiation by a method of spin coefficients, J. Math. Phys. (N.Y.) 3, 566 (1962).

[4] R. M. Wald, General Relativity (University of Chicago Press, Chicago, 1984).

[5] R. Penrose, Zero rest mass fields including gravitation: Asymptotic behavior, Proc. R. Soc. A 284, 159 (1965).

[6] T. Damour, Analytical calculations of gravitational radiation, in 4th Marcel Grossmann Meeting on General Relativity, edited by R. Ruffini (North-Holland, Amsterdam, 1986), pp. 365-392.

[7] D. Christodoulou, The Global Initial Value Problem in General Relativity (World Scientific, Singapore, 2002), pp. 44-54.

[8] D. Christodoulou and S. Klainerman, The Global Nonlinear Stability of the Minkowski Space (Princeton University Press, Princeton, 1993).

[9] J. Bicák, D. Lynden-Bell, and C. Pichon, Relativistic discs and flat galaxy models, Mon. Not. R. Astron. Soc. 265, 126 (1993).

[10] L. Andersson and P. T. Chruściel, Hyperboloidal Cauchy Data for Vacuum EInstein Equations and Obstructions to Smoothness of Null Infinity, Phys. Rev. Lett. 70, 2829 (1993).

[11] L. Andersson and P.T. Chruściel, On "hyperboloidal" Cauchy data for vacuum Einstein equations and obstructions to smoothness of Scri, Commun. Math. Phys. 161, 533 (1994).

[12] P. T. Chruściel, M. A. MacCallum, and D. B. Singleton, Gravitational waves in general relativity XIV. Bondi expansions and the "polyhomogeneity" of Scri, Phil. Trans. R. Soc. A 350, 113 (1995).
[13] A. Ashtekar, J. Bičák, and B. G. Schmidt, Behavior of Einstein-Rosen waves at null infinity, Phys. Rev. D 55, 687 (1997).

[14] H. Friedrich, Peeling or not peeling-Is that the question?, Classical Quantum Gravity 35, 083001 (2018).

[15] Y. Angelopoulos, S. Aretakis, and D. Gajic, Late-time asymptotics for the wave equation on spherically symmetric, stationary spacetimes, Adv. Math. 323, 529 (2018).

[16] Y. Angelopoulos, S. Aretakis, and D. Gajic, Logarithmic corrections in the asymptotic expansion for the radiation field along null infinity, J. Hyperbol. Differ. Equations 16, 1 (2019).

[17] P. T. Chrusciel, J. Jezierski, and M. A. MacCallum, Uniqueness of the Trautman-Bondi mass, Phys. Rev. D 58, 084001 (1998).

[18] J. A. Valiente-Kroon, Conserved quantities for polyhomogeneous spacetimes, Classical Quantum Gravity 15, 2479 (1998).

[19] J. A. Valiente-Kroon, Logarithmic Newman-Penrose constants for arbitrary polyhomogeneous spacetimes, Classical Quantum Gravity 16, 1653 (1999).

[20] H. Godazgar, M. Godazgar, and C. Pope, Subleading BMS charges and fake news near null infinity, J. High Energy Phys. 01 (2019) 143.

[21] H. Godazgar, M. Godazgar, and C. Pope, Tower of subleading dual BMS charges, J. High Energy Phys. 03 (2019) 057.

[22] E. T. Newman and R. Penrose, New conservation laws for zero rest-mass fields in asymptotically flat space-time, Proc. R. Soc. A 305, 175 (1968).

[23] V. Iyer and R. M. Wald, Some properties of the Noether charge and a proposal for dynamical black hole entropy, Phys. Rev. D 50, 846 (1994).

[24] G. Barnich and F. Brandt, Covariant theory of asymptotic symmetries, conservation laws and central charges, Nucl. Phys. B633, 3 (2002).

[25] G. Barnich and C. Troessaert, BMS charge algebra, J. High Energy Phys. 12 (2011) 105.

[26] H. Godazgar, M. Godazgar, and C. Pope, New dual gravitational charges, Phys. Rev. D 99, 024013 (2019). 
[27] H. Godazgar, M. Godazgar, and M. J. Perry, Asymptotic Gravitational Charges, Phys. Rev. Lett. 125, 101301 (2020).

[28] H. Godazgar, M. Godazgar, and M. J. Perry, Hamiltonian derivation of dual gravitational charges, arXiv:2007.07144.

[29] J. A. Valiente-Kroon, A comment on the outgoing radiation condition for the gravitational field and the peeling theorem, Gen. Relativ. Gravit. 31, 1219 (1999).

[30] G. Compère and A. Fiorucci, Advanced lectures on general relativity, arXiv:1801.07064.
[31] R. M. Wald and A. Zoupas, General definition of "conserved quantities" in general relativity and other theories of gravity, Phys. Rev. D 61, 084027 (2000).

[32] H. Godazgar, M. Godazgar, and C. Pope, Dual gravitational charges and soft theorems, J. High Energy Phys. 10 (2019) 123.

[33] J. N. Goldberg, A. J. MacFarlane, E. T. Newman, F. Rohrlich, and E. G. Sudarshan, Spin-s spherical harmonics and edth, J. Math. Phys. (N.Y.) 8, 2155 (1967). 